hep-lat/9604012

ESI (1995) 299

SU-4240-621

DFTUZ/96/06

DSF-T-15/96

UICHEP-TH/95-12

April 1996, Revised December 1996

\title{
Lattice Gauge Fields and Noncommutative Geometry
}

\author{
A.P. Balachandran ${ }^{1}$, G. Bimonte ${ }^{2}$ G. Landi $^{3,4}$, \\ F. Lizzi ${ }^{4,5}$, P. Teotonio-Sobrinho ${ }^{6}$ 用 \\ The E. Schrödinger International Institute for Mathematical Physics, \\ Pasteurgasse 6/7, A-1090 Wien, Austria. \\ 1 Department of Physics, Syracuse University, Syracuse, NY 13244-1130, USA. \\ 2 Departamento de Fisica Teorica, Facultad de Ciencias, \\ Universitad de Zaragoza, 50009 Zaragoza, Spain. \\ 3 Dipartimento di Scienze Matematiche, Università di Trieste, \\ P.le Europa 1, I-34127, Trieste, Italy. \\ 4 INFN, Sezione di Napoli, Napoli, Italy. \\ 5 Dipartimento di Scienze Fisiche, Università di Napoli, \\ Mostra d' Oltremare, Pad. 19, I-80125, Napoli, Italy. \\ ${ }^{6}$ Department of Physics, University of Illinois at Chicago, \\ Chicago, IL 60607-7059, USA.
}

*Address after 03/01/96: Universidade de Sao Paulo, Instituto de Fisica - DFMA, Caixa Postal 663118, 05389-970 Sao Paulo, SP, Brazil. 


\begin{abstract}
Conventional approaches to lattice gauge theories do not properly consider the topology of spacetime or of its fields. In this paper, we develop a formulation which tries to remedy this defect. It starts from a cubical decomposition of the supporting manifold (compactified spacetime or spatial slice) interpreting it as a finite topological approximation in the sense of Sorkin. This finite space is entirely described by the algebra of cochains with the cup product. The methods of Connes and Lott are then used to develop gauge theories on this algebra and to derive Wilson's actions for the gauge and Dirac fields therefrom which can now be given geometrical meaning. We also describe very natural candidates for the QCD $\theta$-term and Chern-Simons action suggested by this algebraic formulation. Some of these formulations are simpler than currently available alternatives. The paper treats both the functional integral and Hamiltonian approaches.
\end{abstract}

Keywords: Lattice Gauge Theory. Geometry and topology of complexes. Noncommutative Geometry. Topological Actions. Chern-Simons terms.

1991 MSC: 06B35, 58B30

PACS: 11.15.Ha, 02.40.S, 02.90.+p 


\section{Introduction}

There is general consensus that elementary particle interactions below the scale of 1 Tev are accurately described by the standard model [1]. It is a gauge theory of strong and weak interactions based on the hypothesis that spacetime is a manifold. Strong processes and weak phenomenology give persuasive evidence that it is a good model at these energies, at least whenever perturbation theory gives a decent reliable approximation.

Nevertheless several central issues in elementary particle physics cannot be addressed using perturbation theory. Examples are problems concerning the bound state spectrum, quark confinement and chiral symmetry breaking in QCD. Numerical schemes, known generically as lattice gauge theories [2], have been developed during the past several years because of limitations of perturbation theory and other analytical methods, and much effort has also been spent studying QCD with their help.

In previous work [3, 4, 5], we have emphasized that conventional formulations of lattice gauge theories are naive in their treatment of topology. They typically begin from an approximation of the supporting manifold (spacetime or spatial slice) with a finite set of points having the trivial discrete topology (each point being both open and closed), and with no further structure. This rough and crude attitude precludes transparent and convincing representations of continuum topology. In fact, it even provokes the suspicion that lattice models are incapable of describing the continuum in a good manner at any finite level of approximation.

This paper attempts to remedy this defect. It starts with the observation that cell decompositions of the manifold, such as cubical and simplicial ones, are associated with certain open covers which in our context can also be realistically assumed to be finite. They are thus finite topological spaces, being examples of partially ordered sets or posets of Sorkin [6, 7, 8]. The latter have been treated in detail elsewhere and shown capable of reproducing subtle continuum features significant for quantum physics [6, 3, 5].

Now the chain complex generated by cells are "dual" to an associative noncommutative differential algebra $\Omega^{*} \mathcal{A}$ which encodes all the information in the chains and can reproduce the latter in full detail. The algebra $\Omega^{*} \mathcal{A}$ is just a variant of the algebra of cochains under the cup product 90 17. It is even possible to reproduce the poset from its differential ideals just as a Hausdorff space can be reproduced from its $C^{*}$-algebra of continuous functions using the Gel'fand-Naimark construction [12]. In this manner, we encode the topology of the approximating set in an algebra.

There are distinct advantages to working with $\Omega^{*} \mathcal{A}$ instead of the chains because of the beautiful methods from Connes, Lott and others [13, 14, 15] for writing gauge theories starting from algebras. By adapting them to $\Omega^{*} \mathcal{A}$, we are able to show that exactly the Wilson actions [2] for the glue and Fermi fields are reproduced by these methods

${ }^{\dagger}$ A previous approach using a nonassociative modification of the algebra of cochains for lattice gauge theories is described in [10]. Nonassociative algebras like those in 10 have also been used in 11] to develop discrete topological field theories. 
for a cubical chain complex. These actions thus naturally emerge from an approach to discretisation which pays conscious attention to the continuum topology.

The action of QCD has a topological term, namely the integral of $\frac{\theta}{8 \pi^{2}} \operatorname{Tr} F \wedge F$, with $F$ being the curvature. This " $\theta$-term", or topological susceptibility is 'topological' since it is independent of spacetime metric. The "strong CP problem" is due to its existence. Its integrand also governs the chiral anomaly and has a central role in studies of chiral symmetry breakdown, the $U(1)$ problem and decays like $\pi^{0} \rightarrow 2 \gamma$. There are thus numerous reasons for finding a lattice analogue of the $\theta$-term. Several lattice versions have also been proposed [16, 22, 17, 18, but none is truly natural. The algebraic approach too has a suggestion for this term. It is very natural, being unique, and involves fewer Wilson links than existing proposals. In addition, the algebraic approach has natural discrete versions for the topological term of two-dimensional QED and also the Chern-Simons [19] term. [For previous discretisations of the latter see [20, 21].]

In what follows, we will be discussing these topological terms in the context of the algebraic approach. Exploration of their properties by lattice theorists is indicated, as they may be viable substitutes to existing versions.

The paper is organized as follows. Section 2 is a short introduction to Sorkin's posets [6] and their relation to chain decompositions of manifolds. Section 3 discusses the cochain algebra while Section 4 formulates integration theory using this algebra. Sections 5 to 10 limit themselves to $\Omega^{*} \mathcal{A}$ for cubical decompositions. Section 5 introduces the algebra $\Omega^{*} \mathcal{A}$ and applies the ideas of Connes and Lott [13, 14, 15] to show the emergence of the Wilson action in a simple way from the algebraic approach. Section 6 does the same to derive Wilson's fermion action from the algebraic approach. Section 7 studies the $2 \mathrm{~d}$ topological action while Sections 8 and 9 extend this discussion to the four dimensional case, and to CS terms in one and three dimensions. The material in Sections 5 to 9 is in the context of functional integrals, as they deal with actions to be fed into these integrals. In Section 10, we also sketch how a Hamiltonian formulation can be derived taking advantage of an earlier work of Rajeev 222. The final Section 11 outlines possible generalizations of the algebraic approach to simplicial and other discretisations of the supporting manifold. They can be relevant because cubical decompositions are convenient only for toroidal topologies, and so for spacetime or spatial manifolds compactified with periodic boundary conditions. In addition this section speculates about the reason why the QCD $\theta$ is small and also points out the advantages of the algebraic approach in subjects like soliton physics, which are not gauge theories.

In previous work $4,5,23$, we had associated an algebra $\mathcal{A}$, different from $\Omega^{*} \mathcal{A}$, with Sorkin's topological approximations. That algebra was $C^{*}$ unlike $\Omega^{*} \mathcal{A}$. It was also infinite -dimensional creating problems of interpretation and adaptation to numerical use. Although not a $C^{*}$-algebra,$\Omega^{*} \mathcal{A}$ does not have these problems and therefore may be superior to $\mathcal{A}$. 


\section{Noncommutative Lattices and Chains}

In this Section we describe the topological approximation method for topological spaces developed previously by Sorkin [6]. We will show that a finite (or finitary) approximation to a topological space can be described by a poset. This poset with its topology is the structure space of a noncommutative algebra. Hence the name Noncommutative Lattices, which we use interchangeably with the word poset.

\section{$2.1 \quad$ Posets}

The word poset [24] is an acronym for a "partially ordered set". We will encounter only finite posets, so we assume that all our posets are finite. Generalization to the countable case is straightforward. If $P=\left\{p_{1}, p_{2}, \ldots, p_{K}\right\}$ is a poset with $K$ elements, then by definition, there is an order relation $\preceq$ between some pairs of its points such that $i$ ) $p_{i} \preceq p_{j}$ and $p_{j} \preceq p_{k} \Rightarrow p_{i} \preceq p_{k}$ (transitivity) and $\left.i i\right) p_{i} \preceq p_{i}$.

A poset has a canonical topology derived from its partial order. A basis of open sets $O_{j}$ for this topology is as follows : the smallest open set $O_{j}$ containing $p_{j}$ consists of all $p_{k}$ such that $p_{k} \preceq p_{j}$ :

$$
O_{j}=\left\{p_{k}: p_{k} \preceq p_{j}\right\}
$$

Sorkin [6] has shown that every finite open cover of a manifold $M$ leads to a (finite) poset. Also a simplicial or cubical decomposition leads to a poset. This result will be illustrated below. It has also been shown [6, 23] that $M$ can be recovered as a topological space from a repeated refinement of the covers and a suitable inverse limit.

\subsection{The Circle and its Noncommutative Lattice}

Let us work out the example of the circle in some detail. The circle $S^{1}$ can be discretised using the lattice $L_{N}$ with $N$ points having coordinates $z_{k}=e^{i k \frac{2 \pi}{N}}, k \in \mathbb{Z} \bmod N$. This lattice gives rise to a cubical decomposition of $S^{1}$ with zero and one cells

$$
C_{0}=\left\{z_{0}, z_{1}, \ldots, z_{N-1}\right\} ; \quad C_{1}=\left\{z_{0,1}, z_{1,2}, \ldots, z_{N-1, N}\right\}
$$

where $z_{j, j+1}:=$ the interval $\left(z_{j}, z_{j+1}\right)$. The elements of the vector space over $\mathbb{C}$ freely generated by these cells are the chains. We can also introduce a boundary operator $\partial$ on these chains according to

$$
\partial z_{j}=0, \quad \partial z_{j, j+1}=z_{j+1}-z_{j}
$$

$\partial^{2}$ being consequently zero. Here $z_{j}$ and $z_{j+1}$ are to be regarded as the boundaries of $z_{j, j+1}$. The chains become a chain complex in the presence of $\partial$. 
There is a natural noncommutative lattice associated with $C_{0}$ and $C_{1}$. We set $z_{j} \preceq z_{j}$, $z_{j, j+1} \preceq z_{j, j+1}$ and $z_{k, k+1} \preceq z_{j}$ if $j \in\{k, k+1\}$. The resultant noncommutative lattice can be conveniently shown with the aid of a Hasse diagram [24] as in Fig. 1. In this diagram, if two points $a$ and $b$ are connected by a link, and $b$ is lower than $a$, then $b \preceq a$.

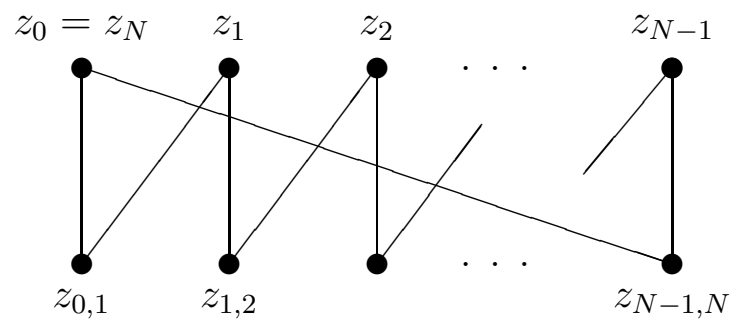

Fig. 1. The Hasse diagram of the circle poset.

In Hasse diagrams with many levels, the corresponding statement is as follows. If $\xi_{0}$ and $\xi_{L}$ are two points in the diagram and it has points $\xi_{1}, \xi_{2}, \ldots, \xi_{L-1}$ such that $\xi_{i}$ and $\xi_{i+1}$ are linked and $\xi_{i}$ is lower than $\xi_{i+1}$, then $\xi_{0} \preceq \xi_{L}$. See Fig. 2 for the example of a two sphere poset taken from Sorkin [6]. Note that the partial order rule of the two-level diagram plus transitivity gives the rule for any diagram.

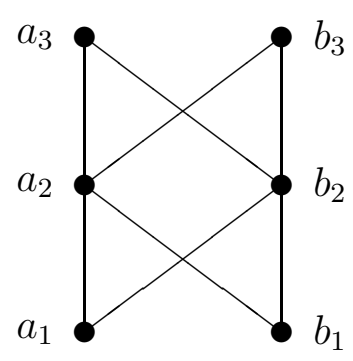

Fig. 2. A two-sphere poset. Here $a_{j} \preceq a_{k}$ or $b_{k}, b_{j} \preceq a_{k}$ or $b_{k}$ whenever $j<k$.

According to poset theory, the basis of open sets for the poset $P$ of Fig. 1 consists of the following:

$$
O_{j, j+1}=\left\{z_{j, j+1}\right\}, \quad O_{j}=\left\{z_{j}, z_{j-1, j}, z_{j, j+1}\right\} .
$$

What is the relation of these open sets to those of $S^{1}$ ? 
There is in fact a natural correspondence between these open sets. Let us assume that $z_{j, j+1}$, represents the open interval $\left\{e^{i \theta \frac{2 \pi}{N}}: j<\theta<j+1\right\}$ of $S^{1}$. Then one sees right away that $O_{j}$ and $O_{j, j+1}$ are open in $S^{1}$. More pedantically, we can construct a map $\varphi: S^{1} \rightarrow P$ by setting $\varphi(p)=p$ if $p=z_{i}$ and $\varphi(p)=z_{j, j+1}$ if $p \in z_{j, j+1}$. Then $\varphi^{-1}\left(O_{j}\right)$ and $\varphi^{-1}\left(O_{j, j+1}\right)$ are open or $\varphi$ is continuous. The topology of $P$ is in fact the quotient topology induced by the map $\varphi$.

One further point may be made. The open sets $\varphi^{-1}\left(O_{j}\right)$ and $\varphi^{-1}\left(O_{j, j+1}\right)$ provide an open cover of $S^{1}$. Suppose now that we identify any two points if neither or both are in every one of these open sets. Then we get back $P, \varphi$ being just this identification map. This construction of $P$ from an open cover is an example of Sorkin's construction [6] of a poset from a manifold $M$.

The interpretation of $P$ as a topological approximation of $M$ has been successfully made by Sorkin [6]. The physical meaning and mathematical power of this approximation have been dealt with elsewhere [6, 23, 3, $1,0,5,25]$. It is in this way that topological data of $M$ are stored in its chains.

These considerations easily extend to any chain complex, such as a simplicial complex (obtained from a simplicial decomposition) of a generic manifold $M$. Thus supposing that

$\alpha_{(n)}^{l}$ denotes an $n$-cell, the partial order is introduced by setting $\alpha_{(m+1)}^{k} \preceq \alpha_{(m)}^{j}$ if $\alpha_{(m)}^{j}$ is a face of $\alpha_{(m+1)}^{k}$. The resultant poset $P$ is a topological space.

If now we regard each cell as consisting only of its interior points, then the open sets of $P$ lead as before to an open cover of $M$. The identification map $\varphi$ from $M$ to $P$ can also be constructed as we did above, the poset topology being just the quotient topology induced by $\varphi$.

Thus always the cells of a manifold $M$ and their incidence relations [26] define a finite topological space $P$ approximating $M$ including its topology.

\section{The Cup Algebra}

In this section we define a differential calculus for forms and chains on noncommutative lattices, based on the cup product. Differential calculi on noncommutative spaces have been discussed elsewhere [27] in different contexts.

\subsection{Construction}

In the usual way [26, we associate a complex vector space of chains with the preceding cells by taking their complex linear combinations. We also define a boundary operator $\partial$ 
such that $\partial^{2}=0$ :

$$
\begin{gathered}
\partial \alpha_{(m)}^{j}=\sum_{k} I\left(\alpha_{(m)}^{j}, \alpha_{(m-1)}^{k}\right) \alpha_{(m-1)}^{k}, \quad I\left(\alpha_{(m)}^{j}, \alpha_{(m-1)}^{k}\right)= \pm 1 \text { or } 0 \\
\partial^{2}=0 \quad \text { or } \quad \sum_{k} I\left(\alpha_{(m)}^{j}, \alpha_{(m-1)}^{k}\right) I\left(\alpha_{(m-1)}^{k}, \alpha_{(m-2)}^{l}\right)=0 .
\end{gathered}
$$

Here $I\left(\alpha_{(m)}^{j}, \alpha_{(m-1)}^{k}\right)$ are incidence numbers. The chain complex we have here will be called $\mathcal{C}$.

Let $\mathcal{C}^{*}$ be the vector space dual to $\mathcal{C}$. It has a basis $\alpha_{j}^{(n)}$ defined by

$$
\left\langle\alpha_{j}^{(m)}, \alpha_{(n)}^{k}\right\rangle=\delta^{m}{ }_{n} \delta_{j}^{k}
$$

$\langle\cdot, \cdot\rangle$ being the duality pairing.

The dual $d$ of $\partial$ is given by the relation

$$
\left\langle d \alpha_{j}^{(m)}, \alpha_{(n)}^{k}\right\rangle=\left\langle\alpha_{j}^{(m)}, \partial \alpha_{(n)}^{k}\right\rangle .
$$

It is nilpotent:

$$
d^{2}=0
$$

The cochain complex $\mathcal{C}^{*}$ can be made into an algebra by defining a product of its elements. This product is the cup product 90 which is the discrete analogue of the wedge product on differential forms. It can be introduced as follows. One first orders the vertices of $\mathcal{C}$ by numbering them from 0 to $N-1$. Any oriented cell is given by its ordered vertices, an odd permutation of the order changing its sign. It can therefore be written as $\left[i_{0}, i_{1}, \ldots, i_{k}\right]=(-1)^{P}\left[j_{0}, j_{1}, \ldots, j_{k}\right]$ where $j_{0}<j_{1}<\ldots<j_{k}$ and $(-1)^{P}$ is the sign of the permutation $P$ bringing the entries on the left to those on the right. We can then label the elements of the dual basis by $\left[j_{0}, j_{1}, \ldots, j_{k}\right]^{*}=(-1)^{P}\left[i_{0}, i_{1}, \ldots, i_{k}\right]^{*},\left[j_{0}, j_{1}, \ldots, j_{k}\right]^{*}$ giving 1 on pairing with $\left[j_{0}, j_{1}, \ldots, j_{k}\right]$ and zero with a cell having any vertex different.

With this convention at hand, the cup product $\sqcup$ is defined as follows:

$$
\left[a_{0}, a_{1}, \ldots, a_{k}\right]^{*} \sqcup\left[b_{0}, b_{1}, \ldots, b_{l}\right]^{*}
$$

is a cochain nonvanishing only on a cell with $k+l+1$ vertices. So we must give its value on $\left[c_{0}, c_{1}, \ldots, c_{k+l}\right]$. We first write the last as $\epsilon\left[d_{0}, d_{1}, \ldots, d_{k+l}\right]$ where $\epsilon$ is 1 or -1 and $d_{0}<d_{1}<\ldots<d_{k+l}$. Then

$$
\begin{gathered}
\left\langle\left[a_{0}, a_{1}, \ldots, a_{k}\right]^{*} \sqcup\left[b_{0}, b_{1}, \ldots, b_{l}\right]^{*},\left[c_{0}, c_{1}, \ldots, c_{k+l}\right]\right\rangle= \\
=\epsilon\left\langle\left[a_{0}, a_{1}, \ldots, a_{k}\right]^{*},\left[d_{0}, d_{1}, \ldots, d_{k}\right]\right\rangle\left\langle\left[b_{0}, b_{1}, \ldots, b_{l}\right]^{*},\left[d_{k}, d_{k+1}, \ldots, d_{k+l}\right]\right\rangle .
\end{gathered}
$$

The cup product is associative.

We call the (associative) algebra of cochains under the cup product as $\omega^{*} \mathcal{A}$, reserving the symbol $\Omega^{*} \mathcal{A}$ for its modifications, to be introduced later. The ordering of vertices, 
and hence $\sqcup$ not being unique, $\omega^{*} \mathcal{A}$ is also not unique. It does become unique when projected down to cohomology classes.

The algebra $\mathcal{A}$, wherefrom the notation $\omega^{*} \mathcal{A}$ is derived, consists of zero cochains and is abelian. We also denote it by $\omega^{(0)} \mathcal{A}, \omega^{(k)} \mathcal{A}$ signifying $k$-cochains, non-vanishing only on chains with $(k+1)$ vertices. Thus $\omega^{*} \mathcal{A}$ has a natural grading, being $\oplus_{k} \omega^{(k)} \mathcal{A}$, with $\left(\omega^{(k)} \mathcal{A}\right)\left(\omega^{(l)} \mathcal{A}\right) \subseteq \omega^{(k+l)} \mathcal{A}$. The operator $d$ is of grade or degree 1 since $d \omega^{(k)} \mathcal{A} \subseteq \omega^{(k+1)} \mathcal{A}$.

The elements of $\omega^{(k)} \mathcal{A}$ can be legitimately regarded as approximations to differential $k$-forms, and $\omega^{*} \mathcal{A}$ as approximating the algebra of differential forms. But there is one notable difference: in general

$$
\alpha^{(k)} \alpha^{(l)} \neq(-1)^{k l} \alpha^{(l)} \alpha^{(k)}, \quad \alpha^{(k)} \in \omega^{(k)} \mathcal{A}, \alpha^{(l)} \in \omega^{(l)} \mathcal{A} .
$$

In other words, $\omega^{*} \mathcal{A}$ is not graded-commutative in contrast to the algebra of differential forms. This fact will become a source of unpleasantness when formulating gauge theories.

In analogy to usage for manifolds, we shall call the elements of $\omega^{(k)} \mathcal{A}$ as $k$-forms.

\subsection{Examples}

Let us look at cubical decomposition of tori as examples.

We begin with $S^{1}$ and the lattice $L_{N}$. The algebra $\mathcal{A}$ then consists of functions on $L_{N}$ with the basis $\left\{z^{j}\right\}$ defined by

$$
\left\langle z^{j}, z_{k}\right\rangle=\delta_{k}^{j}
$$

The one-forms give the $\mathcal{A}$-module generated by the duals $z^{i, i+1}$ of $z_{i, i+1}$ defined by $\left\langle z^{i, i+1}, z_{j, j+1}\right\rangle=\delta^{i}{ }_{j}$. There are no forms of larger order. Of course the definition of the $\mathcal{A}$-module involves the cup product. For the order given by the subscripts on $z_{j}$ 's, one verifies it to be

$$
\begin{aligned}
z^{j} \sqcup z^{k} & =\delta_{j k} z^{j}, \\
z^{k} \sqcup z^{j, j+1} & =\delta_{j k} z^{j, j+1}, \\
z^{j, j+1} \sqcup z^{k} & =\delta_{j+1, k} z^{j, j+1}, \\
z^{j, j+1} \sqcup z^{k, k+1} & =0,
\end{aligned}
$$

where, as would be expected, indices differing by $N$ are to be identified.

These product rules can be written in terms of components of elements. Two general elements of the algebra can be written as

$$
\begin{aligned}
\alpha & =\sum \alpha_{i} z^{i}+\sum \alpha_{i, i+1} z^{i, i+1}, \\
\beta & =\sum \beta_{k} z^{k}+\sum \beta_{k, k+1} z^{k, k+1},
\end{aligned}
$$


so that coordinate representations of $\alpha$ and $\beta$ are

$$
\begin{aligned}
\alpha & =\left(\alpha_{0}, \alpha_{1}, \ldots, \alpha_{N-1} ; \alpha_{0,1}, \alpha_{1,2}, \ldots \alpha_{N-1, N}\right) \\
\beta & =\left(\beta_{0}, \beta_{1}, \ldots, \beta_{N-1} ; \beta_{0,1}, \beta_{1,2}, \ldots \beta_{N-1, N}\right)
\end{aligned}
$$

while the cup product can also be written as

$$
\begin{aligned}
\alpha \beta= & \left(\alpha_{0} \beta_{0}, \alpha_{1} \beta_{1}, \cdots, \alpha_{N-1} \beta_{N-1} ;\right. \\
& \left.\alpha_{0} \beta_{0,1}+\alpha_{0,1} \beta_{1}, \alpha_{1} \beta_{1,2}+\alpha_{1,2} \beta_{2}, \cdots, \alpha_{N-1} \beta_{N-1, N}+\alpha_{N-1, N} \beta_{N}\right) .
\end{aligned}
$$

The (left) regular representation of this algebra in the basis $\left\{z^{01}, z^{12}, \ldots, z^{N-1, N} ; z^{0}, z^{1}, \ldots, z^{N-1}\right\}$ is

$$
\begin{aligned}
& \sum \alpha_{j} z^{j} \rightarrow \operatorname{diag}\left[\alpha_{0}, \alpha_{1}, \ldots, \alpha_{N-1} ; \alpha_{0}, \alpha_{1}, \ldots, \alpha_{N-1}\right] \\
& \sum \alpha_{j, j+1} z^{j, j+1} \rightarrow\left[\begin{array}{cccccccc} 
& 0 & \alpha_{0,1} & 0 & \cdot & \cdot & \cdot & 0 \\
0 & 0 & 0 & \alpha_{1,2} & 0 & & & 0 \\
& \cdot & \cdot & 0 & \cdot & & \cdot \\
0 & 0 & \cdot & \cdot & \cdot & 0 & \alpha_{N-2, N-1} \\
0 & \alpha_{N-1,0} & 0 & \cdot & \cdot & \cdot & 0 & 0
\end{array}\right] .
\end{aligned}
$$

Now, $\omega^{*} \mathcal{A}$ is a differential algebra with a differential $d$ which is the dual of the following boundary operator $\partial$ on chains:

$$
\begin{aligned}
\partial z_{j} & =0 \\
\partial z_{j, j+1} & =z_{j+1}-z_{j}, \\
\partial^{2} & =0
\end{aligned}
$$

It is given by

$$
\begin{aligned}
d z^{j} & =z^{j-1, j}-z^{j, j+1}, \\
d z^{j, j+1} & =0,
\end{aligned}
$$

so that $d^{2}=0$.

It turns out that for cubical decompositions, $d$ can be realized in terms of a linear operator $F_{+}$in the regular representation, much as in cyclic cohomology [14, the relevant formula being

$$
d \alpha^{(k)}=F_{+} \alpha^{(k)}-(-1)^{k} \alpha^{(k)} F_{+},
$$

where we have identified $\omega^{*} \mathcal{A}$ with its representation. In fact $F_{+} \in \omega^{(1)} \mathcal{A}$. For the present case of $S^{1}$, the formula for $F_{+}$is

$$
F_{+}=\left(\begin{array}{cc}
0 & \Delta \\
0 & 0
\end{array}\right), \quad \Delta_{r s}=\delta_{r, s-1}
$$


It may be remarked that

$$
\Delta\left(\begin{array}{ccc}
\alpha_{0} & & \\
& \ddots & \\
& & \alpha_{N-1}
\end{array}\right) \Delta^{\dagger}=\left(\begin{array}{ccc}
\alpha_{1} & & \\
& \ddots & \\
& & \alpha_{N}
\end{array}\right) ; \alpha_{N}=\alpha_{0},
$$

that is, it consists of $\alpha_{i}$ rotated by $e^{+i 2 \pi / N}$. We have the identities

$$
\begin{aligned}
\Delta^{\dagger} & =\Delta^{T}=\Delta^{-1}, \\
\Delta^{N} & =I I, \operatorname{det} \Delta=(-1)^{N-1} .
\end{aligned}
$$

Later on, we will modify this algebra to another one, more appropriate for gauge theories, to work with.

Note that

$$
F=\left(\begin{array}{cc}
0 & \Delta \\
\Delta^{\dagger} & 0
\end{array}\right), \widetilde{F}=i\left(\begin{array}{cc}
0 & -\Delta \\
\Delta^{\dagger} & 0
\end{array}\right) \quad \text { and } \gamma=\left(\begin{array}{cc}
I I & 0 \\
0 & -I I
\end{array}\right)
$$

generate a Clifford algebra (isomorphic to that of Pauli matrices) and that $F_{+}=\frac{1}{2}(F+i \widetilde{F})$ anti-commutes with $\gamma$. Furthermore

$$
F_{+}^{2}=0
$$

Generalization to higher dimensions is easy. Consider for example $S^{1} \times S^{1}:=T^{2}$. We can assume that its cubical cells are given by the Cartesian product $L_{N} \times L_{N}$. As for its differential algebra, first let

$$
\omega^{*} \mathcal{A}^{(1)}:=\omega^{*} \mathcal{A} \otimes_{\mathbf{C}} \text { II , }
$$

$\omega^{*} \mathcal{A}$ being for $L_{N}$ and II being the constant function with value 1 . The algebra $\omega^{*} \mathcal{A}^{(1)}$ will consist of elements describing the first $L_{N}$ in the product $L_{N} \times L_{N}$. If $\omega^{*} \mathcal{A}^{(2)}$ is to be associated with the second $L_{N}$, its one-forms must anti-commute with those of $\omega^{*} \mathcal{A}^{(1)}$. For this reason, set

$$
\omega^{*} \mathcal{A}^{(2)}=\mathbb{I} \otimes \mathcal{A} \oplus \gamma \otimes \omega^{(1)} \mathcal{A}:=\omega^{(0)} \mathcal{A}^{(2)} \oplus \omega^{(1)} \mathcal{A}^{(2)}:=\mathcal{A}^{(2)} \oplus \omega^{(1)} \mathcal{A}^{(2)} .
$$

Then the algebra of $L_{N} \times L_{N}$, which we again call $\omega^{*} \mathcal{A}$, is generated by $\omega^{*} \mathcal{A}^{(1)}$ and $\omega^{*} \mathcal{A}^{(2)}$,

$$
\omega^{*} \mathcal{A}=\omega^{*} \mathcal{A}^{(1)} \cdot \omega^{*} \mathcal{A}^{(2)} .
$$

The differential $d$ for this algebra is also readily found. Thus let

$$
F_{+}^{(1)}=f_{+} \otimes I I, \quad F_{+}^{(2)}=\gamma \otimes f_{+},
$$

where $f_{+}$is the same matrix which appears in (3.17). [The notation has been slightly changed to avoid confusion below.] Then $d$ is defined by (3.16) where

$$
F_{+}=F_{+}^{(1)}+F_{+}^{(2)}, \quad F_{+} F_{+} \equiv F_{+}^{2}=0 .
$$




\subsection{The Reconstruction Theorem}

There is a nice way to reconstruct the poset $P$ from the algebra $\omega^{*} \mathcal{A}$. It very much resembles the methods used in topologising the structure space (or space of irreducible *-representations (IRR's)) of $C^{*}$-algebras [12] when the former for example is a finite set. We can associate ideals ["primitive ideals"] with points $p$ of the structure space, the ideal $I_{p}$ at $p$ being the kernel of the IRR $p$ [elements which vanish at $p$ ]. There is a distinct $I_{p}$ at distinct $p$. We can now partially order $I_{p}$ by inclusion: $I_{p} \preceq I_{q} \Leftrightarrow I_{p} \subseteq I_{q}$. This gives a partial order also on $p$ 's: $p \preceq q \Leftrightarrow I_{p} \preceq I_{q}$. In this way, the structure space becomes a poset with its topology. The latter is the analogue of the Gel'fand topology for the structure space of abelian $C^{*}$-algebras and equivalent to the hull-kernel topology [12]. As emphasized elsewhere [3, 4, 28], this manner of retrieving topology from algebras seems to be of exceptional importance for foundations of quantum physics.

Now, it is the case that $\omega^{*} \mathcal{A}$ is not a $C^{*}$-algebra, as it does not contain the adjoint of forms of nonvanishing degree. So we must suitably modify the rules of reconstruction of $P$ from its ideals. For this purpose, let us define a differential ideal of $\omega^{*} \mathcal{A}$ as a two-sided ideal closed under $d$. Let us then say that a differential ideal $I$ is indecomposable if it is not the intersection of two other differential ideals $I_{i}$ both distinct from $I: I \neq I_{1} \cap I_{2}$, for $I_{1} \neq I_{2}$ and $I \neq I_{1}$ or $I_{2}$.

If two representations have ideals $I_{1}$ and $I_{2}$, their direct sum has the ideal $I_{1} \cap I_{2}$. Thus the notion of indecomposability here replaces the notion of irreducibility. The latter is not appropriate for us. Our algebra has nilpotent elements and has incompletely reducible faithful representations which we will have to consider.

Let $\left\{I_{\alpha}\right\}$ be the set of indecomposable differential ideals of $\omega^{*} \mathcal{A}$. They can be partially ordered by inclusion just as in the case above:

$$
I_{p} \preceq I_{q} \Leftrightarrow I_{p} \subseteq I_{q}
$$

With this partial order, $\left\{I_{\alpha}\right\}$ becomes a poset which is just the poset $P$.

We content ourselves by verifying this assertion for the circle case. For the latter, the indecomposable differential ideals are

$$
\begin{aligned}
& I_{j}=\left\{\sum \alpha_{k} z^{k}+\sum \alpha_{k, k+1} z^{k, k+1}: \alpha_{j}=0\right\} \\
& I_{j, j+1}=\left\{\sum \alpha_{k} z^{k}+\sum \alpha_{k, k+1} z^{k, k+1}: \alpha_{j}=\alpha_{j, j+1}=\alpha_{j+1}=0\right\} .
\end{aligned}
$$

The proof is by straightforward calculation. Now $I_{j, j+1} \preceq I_{j}$ and $I_{j, j+1} \preceq I_{j+1}$ by the previous rule, so that the poset of these ideals is exactly that of Fig. 1. 


\section{Integration Theory}

A cochain can be evaluated or "integrated" on a chain. We want to express these integrals now using Hilbert spaces traces (partial and otherwise) as in noncommutative geometry [14]-[15], this formulation being very useful for developing gauge theories.

Let us consider the $S^{1}$ case first. Here the pairing of a cochain $\alpha=\sum \alpha_{i} z^{i}+$ $\sum \alpha_{i, i+1} z^{i, i+1}$ with a chain $\xi=\sum \xi^{j} z_{j}+\sum \xi^{j, j+1} z_{j, j+1}$ is

$$
\langle\alpha, \xi\rangle=\sum \alpha_{j} \xi^{j}+\sum \alpha_{j, j+1} \xi^{j, j+1}
$$

We can express this by using matrix elements of operators by setting

$$
\begin{aligned}
& \delta_{j k}=\left\langle z^{j}, z_{k}\right\rangle:=\int_{z_{k}} z^{j} \equiv\left\langle k\left|z^{j}\right| k\right\rangle=\left\langle k+N\left|z^{j}\right| k+N\right\rangle, \\
& \delta_{j k}=\left\langle z^{j, j+1}, z_{k, k+1}\right\rangle:=\int_{z_{k, k+1}} z^{j, j+1} \equiv\left\langle k\left|z^{j, j+1} F_{+}^{\dagger}\right| k\right\rangle,
\end{aligned}
$$

the pairing of zero- (one-) forms with one- (zero-) chains being identified with zero. Here and in what follows, $z^{j}$ and $z^{j, j+1}$ appearing in matrix elements and traces are the operators of (3.13), while $|k>\equiv| k+N>(0 \leq k \leq N-1)$ is the column vector with the row corresponding to the site $e^{i k 2 \pi / N}$ equal to 1 , and the remaining rows zero. Its lower half is in particular zero. From (4.2,4.3), we get the general formula for (4.1) using linearity. Note that the integral of a one-form $\omega$ over the entire circle poset is

$$
\int \omega=\operatorname{Tr} \omega F_{+}^{\dagger}=\sum_{i=1}^{N}\left\langle i\left|\omega F_{+}^{\dagger}\right| i\right\rangle,
$$

while the partial integral from $j$ to $j+s+1$ is

$$
\int_{j}^{j+s+1} \omega=\sum_{i=j}^{j+s}\left\langle i\left|\omega F_{+}^{\dagger}\right| i\right\rangle .
$$

If $\omega$ is exact, that is if $\omega=d \alpha=\left[F_{+}, \alpha\right], \alpha=\sum \alpha_{i} z^{i}$ being a function, then

$$
\int_{j}^{j+s+1} \omega=\alpha_{j+s+1}-\alpha_{j}
$$

so that Stokes' theorem is valid. The manipulations leading to (4.6), are indicated by

$$
\begin{aligned}
\sum_{i=j}^{j+s}\left\langle i\left|\left[F_{+}, \alpha\right] F_{+}^{\dagger}\right| i\right\rangle & =\sum_{i=j}^{j+s}\left[\Delta\left(\begin{array}{cccc}
\alpha_{0} & & & \\
& \alpha_{1} & & \\
& & \ddots & \\
& & & \alpha_{N-1}
\end{array}\right) \Delta^{\dagger}-\left(\begin{array}{cccc}
\alpha_{0} & & & \\
& \alpha_{1} & & \\
& & \ddots & \\
& & \alpha_{N-1}
\end{array}\right)\right]_{i i} \\
& =\alpha_{j+s+1}-\alpha_{j} .
\end{aligned}
$$


It is noteworthy that the traces choose an orientation to the integrals, as we could equally well have expected to get the negatives of the above right-hand sides. It is in fact possible to get the reversed sign with a different cup product. A formalism incorporating both signs can also be developed as we shall later see.

Next consider the two-torus. Its lattice $L_{N} \times L_{N}$ can be labeled by $(\alpha, \beta), \alpha, \beta=$ $0, \ldots, N-1$. On this lattice, we can integrate functions, and one- and two-forms. The general rules of integration can be got from what follows below and by linearity:

i) For functions $z^{(\alpha, \beta)}$,

$$
\left\langle z^{(\alpha, \beta)},\left(\alpha^{\prime}, \beta^{\prime}\right)\right\rangle=\delta_{\left(\alpha, \alpha^{\prime}\right)} \delta_{\left(\beta, \beta^{\prime}\right)}:=\int_{\left(\alpha^{\prime}, \beta^{\prime}\right)} z^{(\alpha, \beta)}=\left\langle\left(\alpha^{\prime}, \beta^{\prime}\right)\left|z^{(\alpha, \beta)}\right|\left(\alpha^{\prime}, \beta^{\prime}\right)\right\rangle .
$$

ii) For the one-form $\omega^{(1)}=\omega \otimes I I$, where $\omega$ is a one-form for the first $L_{N}$, we have

$$
\left\langle\omega^{(1)},((\alpha, \beta),(\alpha+1, \beta))\right\rangle:=\int_{(\alpha, \beta)}^{(\alpha+1, \beta)} \omega^{(1)}=\left\langle(\alpha, \beta)\left|\omega^{(1)} F_{+}^{(1) \dagger}\right|(\alpha, \beta)\right\rangle,
$$

$((\alpha, \beta),(\alpha+1, \beta))$ being the link from $(\alpha, \beta)$ to $(\alpha+1, \beta)$. If, more generally, the one-form is $\omega^{(0)} \omega^{(1)}$, where $\omega^{(0)}$ is a generic function,

$$
\begin{aligned}
\int_{(\alpha, \beta)}^{(\alpha+1, \beta)} \omega^{(0)} \omega^{(1)} & =\left\langle(\alpha, \beta)\left|\omega^{(0)} \omega^{(1)} F_{+}^{(1) \dagger}\right|(\alpha, \beta)\right\rangle \\
& =\omega^{(0)}[(\alpha, \beta)]\left\langle(\alpha, \beta)\left|\omega^{(1)} F_{+}^{(1) \dagger}\right|(\alpha, \beta)\right\rangle, \\
\omega^{(0)}[\alpha, \beta] & \equiv\left\langle\omega^{(0)},(\alpha, \beta)\right\rangle .
\end{aligned}
$$

Similarly, if $\widetilde{\omega}^{(1)}=\gamma \otimes \omega$ is a one-form and $\omega^{(0)}$ a function,

$$
\begin{aligned}
\int_{(\alpha, \beta)}^{(\alpha, \beta+1)} \omega^{(0)} \widetilde{\omega}^{(1)} & =\left\langle(\alpha, \beta)\left|\omega^{(0)} \widetilde{\omega}^{(1)} F_{+}^{(2) \dagger}\right|(\alpha, \beta)\right\rangle \\
& =\omega^{(0)}[(\alpha, \beta)]\left\langle(\alpha, \beta)\left|\widetilde{\omega}^{(1)} F_{+}^{(2) \dagger}\right|(\alpha, \beta)\right\rangle .
\end{aligned}
$$

The integral of $\omega^{(1)}$ over $((\alpha, \beta),(\alpha, \beta+1))$ and of $\widetilde{\omega}^{(1)}$ over $((\alpha, \beta),(\alpha+1, \beta))$ are zero.

iii) Next we come to two-forms. It is enough to consider the integral of a two-form $\omega^{(2)}$ over an elementary cell $\mathcal{C}=((\alpha, \beta),(\alpha+1, \beta)) \times((\alpha, \beta),(\alpha, \beta+1))$. We define

$$
\int_{\mathcal{C}} \omega^{(2)}=\left\langle(\alpha, \beta)\left|\omega^{(2)} F_{+}^{(1)^{\dagger}} F_{+}^{(2)^{\dagger}}\right|(\alpha, \beta)\right\rangle \text {. }
$$

iv) Integrals of $k$-forms over $m$-cells are (of course) zero if $k \neq m$. 
It is important that there are analogues of Stokes' theorems valid in this formulation. Consider first the integral of an exact one-form $d \omega^{(0)}$ over a series of touching links. Applying (4.6) to each link successively, we find Stokes' theorem for one-forms.

A corollary of this result is that the integral of an exact form over a closed loop of links is zero. It means also that its integrals from $A$ to $B$ by any two paths are equal, that is, "continuous deformation of paths keeping ends fixed" does not change the integral.

Finally, consider an exact two-form $d \omega=F_{+} \omega+\omega F_{+}, \omega$ being a one-form. Its Stokes' theorem for the elementary cell $\mathcal{C}$ would read

$$
\int_{\mathcal{C}} d \omega=\int_{\partial \mathcal{C}} \omega
$$

The proof is as follows. For the right-hand side, from (4.94.12) one gets

$$
\begin{aligned}
\int_{\partial \mathcal{C}} \omega= & \left\langle(\alpha, \beta)\left|\omega F_{+}^{(2)^{\dagger}}\right|(\alpha, \beta)\right\rangle+\left\langle(\alpha, \beta+1)\left|\omega F_{+}^{(1)^{\dagger}}\right|(\alpha, \beta+1)\right\rangle \\
& -\left\langle(\alpha, \beta)\left|\omega F_{+}^{(1)^{\dagger}}\right|(\alpha, \beta)\right\rangle-\left\langle(\alpha+1, \beta)\left|\omega F_{+}^{(2)^{\dagger}}\right|(\alpha+1, \beta)\right\rangle .
\end{aligned}
$$

Now, $\omega$ has the general form $\omega=\xi f_{+} \otimes I I+\eta \gamma \otimes f_{+}$, where $\xi, \eta$ are functions, while, from (3.25), $F_{+}^{(1)}=f_{+} \otimes I I, F_{+}^{(2)}=\gamma \otimes f_{+}$. In view of this, we can write (4.15) as

$$
\begin{aligned}
\int_{\partial \mathcal{C}} \omega & =\langle(\alpha, \beta)|\eta|(\alpha, \beta)\rangle+\langle(\alpha, \beta+1)|\xi|(\alpha, \beta+1)\rangle \\
& -\langle(\alpha, \beta)|\xi|(\alpha, \beta)\rangle-\langle(\alpha+1, \beta)|\eta|(\alpha+1, \beta)\rangle .
\end{aligned}
$$

For the left-hand side, by writing $\xi=\sum_{j} \xi_{j}^{(1)} \otimes \xi_{j}^{(2)}$ and $\eta=\sum_{j} \eta_{j}^{(1)} \otimes \eta_{j}^{(2)}$, one finds that

$$
\begin{aligned}
\int_{\mathcal{C}} d \omega= & \left\langle(\alpha, \beta)\left|d \omega F_{+}^{(1)^{\dagger}} F_{+}^{(2)^{\dagger}}\right|(\alpha, \beta)\right\rangle \\
= & \left\langle(\alpha, \beta)\left|\left(F_{+} \omega+\omega F_{+}\right) F_{+}^{(1)^{\dagger}} F_{+}^{(2)^{\dagger}}\right|(\alpha, \beta)\right\rangle \\
= & \langle(\alpha, \beta)|\left\{-\sum_{j} f_{+} \eta_{j}^{(1)} f_{+}^{\dagger} \otimes \eta_{j}^{(2)}+\sum_{j} \xi_{j}^{(1)} \otimes f_{+} \xi_{j}^{(2)} f_{+}^{\dagger}\right. \\
& \left.+\sum_{j} \eta_{j}^{(1)} \otimes \eta_{j}^{(2)}-\sum_{j} \xi_{j}^{(1)} \otimes \xi_{j}^{(2)}\right\}|(\alpha, \beta)\rangle .
\end{aligned}
$$

Since $f_{+}=\left(\begin{array}{ll}0 & \Delta \\ 0 & 0\end{array}\right)$, on using (3.18), this becomes

$$
\begin{aligned}
\int_{\mathcal{C}} d \omega= & \left\langle(\alpha, \beta)\left|d \omega F_{+}^{(1)^{\dagger}} F_{+}^{(2)^{\dagger}}\right|(\alpha, \beta)\right\rangle \\
= & -\langle(\alpha+1, \beta)|\eta|(\alpha+1, \beta)\rangle+\langle(\alpha, \beta+1)|\xi|(\alpha, \beta+1)\rangle \\
& +\langle(\alpha, \beta)|\eta|(\alpha, \beta)\rangle-\langle(\alpha, \beta)|\xi|(\alpha, \beta)\rangle,
\end{aligned}
$$


which is seen to coincide with (4.16). Hence Stokes' theorem for $\mathcal{C}$ is true.

In obtaining (4.174.18), we have for instance used the fact that $F_{+}^{(1)} F_{+}^{(1)^{\dagger}}|(\alpha, \beta)\rangle=$ $|(\alpha, \beta)\rangle$ since $|\alpha\rangle$ and $|\beta\rangle$ are column vectors with zero along their last $N$ rows.

Stokes' theorem for a general cube follows by decomposing it into elementary cells like $\mathcal{C}$ and applying (4.15) to each cell.

We can evidently extend these considerations to tori $T^{N}$ of arbitrary dimensions.

\section{Gauge Theories}

\section{$5.1 \quad$ The $*$-Algebra}

It is useful to change the algebra $\omega^{*} \mathcal{A}$ somewhat for physical applications. The reason is that $\omega^{*} \mathcal{A}$ is not a $C^{*}$-algebra and so causes (minor) problems in formulating reality (hermiticity) conditions. Let us first attend to these modifications.

We begin with the circle chains and its algebra (3.9). We enlarge this algebra by introducing new elements $\widetilde{z}^{k, k+1}$ with the properties

$$
z^{j, j+1} \sqcup \widetilde{z}^{k, k+1}=\widetilde{z}^{j, j+1} \sqcup \widetilde{z}^{k, k+1}=0 \text {. }
$$

They are to be thought of as dual to $z_{k, k+1}$, just as $z^{k, k+1}$. But whereas in the definition of $z^{j} \sqcup z^{k, k+1}$, the vertices had the order $[0,1,2, \ldots, N-1]$, we use the order $[N-1, N-$ $2, \ldots, 0]$ in the definition of $z^{j} \sqcup \widetilde{z}^{k, k+1}$, so that

$$
\begin{aligned}
& z^{j} \sqcup \widetilde{z}^{k, k+1}=\widetilde{z}^{k, k+1} \delta_{j, k+1}, \\
& \widetilde{z}^{k, k+1} \sqcup z^{j}=\delta_{j k} \widetilde{z}^{k, k+1} .
\end{aligned}
$$

Representing two elements $\sum \alpha_{j} z^{j}+\sum \alpha_{j, j+1} z^{j, j+1}+\sum \widetilde{\alpha}_{j, j+1} \widetilde{z}^{j, j+1}$ and $\sum \beta_{j} z^{j}+$ $\sum \beta_{j, j+1} z^{j, j+1}+\sum \widetilde{\beta}_{j, j+1} \widetilde{z}^{j, j+1}$ in terms of their components as

$$
\begin{aligned}
& \alpha=\left(\alpha_{0}, \alpha_{1}, \ldots, \alpha_{N-1} ; \alpha_{01}, \alpha_{12}, \ldots \alpha_{N-1, N} ; \widetilde{\alpha}_{01}, \widetilde{\alpha}_{12}, \ldots \widetilde{\alpha}_{N-1, N}\right) \\
& \beta=\left(\beta_{0}, \beta_{1}, \ldots, \beta_{N-1} ; \beta_{01}, \beta_{12}, \ldots \beta_{N-1, N} ; \widetilde{\beta}_{01}, \widetilde{\beta}_{12}, \ldots \widetilde{\beta}_{N-1, N}\right)
\end{aligned}
$$

we now have for their product,

$$
\begin{aligned}
\alpha \beta= & \left(\alpha_{0} \beta_{0}, \alpha_{1} \beta_{1}, \cdots, \alpha_{N-1} \beta_{N-1} ;\right. \\
& \alpha_{0} \beta_{0,1}+\alpha_{0,1} \beta_{1}, \alpha_{1} \beta_{1,2}+\alpha_{1,2} \beta_{2}, \cdots, \alpha_{N-1} \beta_{N-1, N}+\alpha_{N-1, N} \beta_{N} ; \\
& \left.\alpha_{1} \widetilde{\beta}_{0,1}+\widetilde{\alpha}_{0,1} \beta_{0}, \alpha_{2} \widetilde{\beta}_{1,2}+\widetilde{\alpha}_{1,2} \beta_{1}, \cdots, \alpha_{N} \widetilde{\beta}_{N-1, N}+\widetilde{\alpha}_{N-1, N} \beta_{N-1}\right) .
\end{aligned}
$$


The algebra with product (5.4) is a * (but not a $C^{*}-$ ) algebra, the * operation being:

$$
\alpha^{*}=\left(\alpha_{0}^{*}, \alpha_{1}^{*}, \ldots, \alpha_{N-1}^{*} ; \widetilde{\alpha}_{0,1}^{*}, \widetilde{\alpha}_{1,2}^{*}, \ldots \widetilde{\alpha}_{N-1, N}^{*} ; \alpha_{0,1}^{*}, \alpha_{1,2}^{*}, \ldots, \alpha_{N-1, N}^{*}\right)
$$

with the $*$ on the right hand side denoting the usual complex conjugation of complex numbers. This $*$ operation on the algebra fulfills $(\alpha \beta)^{*}=\beta^{*} \alpha^{*}$. We call this algebra as $\Omega^{*} \mathcal{A}$. It may be worth noticing that the reconstruction theorem of Section 3.3 works also for the algebra $\Omega^{*} \mathcal{A}$ if one considers indecomposable differential ideals which are in addition * closed.

A convenient faithful representation of this algebra is as follows. The functions $\mathcal{A}:=$ $\Omega^{(0)} \mathcal{A}$ are diagonal $2 N \times 2 N$ matrices

$$
a=\operatorname{diag}\left[\alpha_{0}, \ldots, \alpha_{N-1} ; \alpha_{0}, \ldots, \alpha_{N-1}\right]
$$

As for one-forms $\Omega^{(1)} \mathcal{A}$, they can be got from

$$
F=\left(\begin{array}{cc}
0 & \Delta \\
\Delta^{\dagger} & 0
\end{array}\right) c,
$$

$c$ being a fermionic annihilation operator. The role of $F$ now is the previous role of $F_{+}$. The one-forms are thus $\sum a_{i}\left[F, b_{i}\right], a_{i}, b_{i} \in \Omega^{(0)} \mathcal{A}$.

Two-forms are zero, $F^{2}$ being zero, the operator $c$ having been inserted for this purpose.

The * operation in this representation is hermitian conjugation on all operators except $c:$

$$
\begin{aligned}
& a^{*}=a^{\dagger} \text { for } a \in \Omega^{(0)} \mathcal{A}, \\
& \left(\sum a_{i}\left[F, b_{i}\right]\right)^{*}=-\sum\left[\left(\begin{array}{cc}
0 & \Delta \\
\Delta^{\dagger} & 0
\end{array}\right), b_{i}^{\dagger}\right] a_{i}^{\dagger} c .
\end{aligned}
$$

Higher dimensional generalizations are easy. For $T^{2}$, we introduce two mutually anticommuting fermion annihilation operators $c_{1}$ and $c_{2}$ and set

$$
\begin{aligned}
F_{1} & =\left[\left(\begin{array}{cc}
0 & \Delta \\
\Delta^{\dagger} & 0
\end{array}\right) \otimes I I\right] c_{1} \\
F_{2} & =\left[I I \otimes\left(\begin{array}{cc}
0 & \Delta \\
\Delta^{\dagger} & 0
\end{array}\right)\right] c_{2}, \\
F & =F_{1}+F_{2} .
\end{aligned}
$$

Then the set of functions for the $T^{2}$-lattice is $\mathcal{A} \otimes \mathbb{C} \mathcal{A}$, one-forms are $\sum f_{i}\left[F, g_{i}\right]$ and twoforms are $\sum f_{i}\left[F, g_{i}\right]\left[F, h_{i}\right],\left(f_{i}, g_{i}, h_{i} \in \mathcal{A} \otimes_{\mathbb{C}} \mathcal{A}\right)$. There are no forms of higher order. The $d$ operator on a form $\omega^{(k)}$ of degree $k$ can be expressed using $F$ :

$$
d \omega^{(k)}=F \omega^{(k)}-(-1)^{k} \omega^{(k)} F .
$$


The integration theory here is similar to that for $\omega^{*} \mathcal{A}$. It is enough to replace $F_{+}^{(i) \dagger}$ by $f_{+}^{(i) \dagger} c_{i}^{\dagger}$ in the appropriate formulas of Section 4 . Here, $f_{+}^{(1)}=f_{+} \otimes \mathbb{I}$ and $f_{+}^{(2)}=\mathbb{I} \otimes f_{+}$ with $f_{+}=\left(\begin{array}{ll}0 & \Delta \\ 0 & 0\end{array}\right)$. [Furthermore, of course traces such as that in (4.4) now involve also traces over creation-annihilation operators.] Note that if $F_{+}^{(i) \dagger}$ are now replaced by $f_{+}^{(i)} c_{i}^{\dagger}$ instead, we will get answers appropriate for integrals with orientations opposite to the previous ones.

For what follows, it is convenient to always call functions as $\mathcal{A}:=\Omega^{(0)} \mathcal{A}$, forms of degree $k$ as $\Omega^{(k)} \mathcal{A}$ and set $\Omega^{*} \mathcal{A}=\oplus_{k} \Omega^{(k)} \mathcal{A}$. This means that we rechristen $\mathcal{A} \otimes_{\mathbb{C}} \mathcal{A}$ above as $\mathcal{A}$. We also will not distinguish $\Omega^{(k)} \mathcal{A}$ and $\Omega^{*} \mathcal{A}$ from their representations.

\subsection{The Yang-Mills Action}

Consider the 'trivial' $\mathcal{A}$-module (the algebraic counterpart of the module of sections of a trivial bundle)

$$
\mathcal{E}=\mathbb{C}^{N} \otimes_{\mathbb{C}} \mathcal{A}:=\mathcal{A}^{N}
$$

Any element of $\mathcal{E}$ will be written as a finite linear combination of 'words'

$$
v \otimes a, \quad v=\left(v_{1}, v_{2}, \ldots, v_{N}\right), v_{i} \in \mathbb{C}, \quad a \in \mathcal{A}
$$

It is a right $\mathcal{A}$-module, with the $\mathcal{A}$ action simply given by $(v \otimes a) b=v \otimes a b$. We also introduce the spaces

$$
\begin{aligned}
& \mathcal{E}^{(k)}:=\mathcal{E} \otimes_{\mathcal{A}} \Omega^{(k)} \mathcal{A}=\mathbb{C}^{N} \otimes_{\mathbb{C}} \Omega^{(k)} \mathcal{A}, \\
& \mathcal{E}^{*}=\oplus_{k} \mathcal{E}^{(k)} .
\end{aligned}
$$

Elements of $\mathcal{E}^{(k)}$ are (finite) combinations of terms like $v^{(k)}=v \otimes \mathbb{C}^{\omega^{(k)}}$, with $v \in \mathbb{C}^{N}$ and $\omega^{(k)} \in \Omega^{(k)} \mathcal{A}$. Any $\mathcal{E}^{(k)}$ is a right $\mathcal{A}$-module while $\mathcal{E}^{*}$ is a right $\Omega^{*} \mathcal{A}$-module.

A connection on the module $\mathcal{E}$ is a linear map $\nabla: \mathcal{E}^{(k)} \rightarrow \mathcal{E}^{(k+1)}$ which satisfies 'Leibnitz rule'

$$
\nabla\left(v^{(k)} \rho\right)=\left(\nabla v^{(k)}\right) \rho+(-1)^{k} v^{(k)} d \rho, \quad \forall v^{(k)} \in \mathcal{E}^{(k)}, \rho \in \Omega^{*} \mathcal{A} .
$$

A gauge potential can be introduced by defining

$$
\nabla=\mathbb{I} \otimes d+T(\alpha) \otimes A(\alpha)
$$

summation on $\alpha$ being understood. The $A(\alpha)$ 's are the connection one-forms and the $T(\alpha)$ 's are matrices (they are taken to be a basis for some Lie algebra, for instance a basis for the Lie algebra $\underline{U}(N)$ of $U(N)$; see later). It is immediate to check that $\nabla$ in (5.15) satisfies (5.14). Explicitly,

$$
\nabla\left(v \otimes \omega^{(k)}\right)=v \otimes d \omega^{(k)}+T(\alpha) v \otimes A(\alpha) \omega^{(k)} .
$$


By the very definition of $\nabla$, one checks that its square is $\Omega^{*} \mathcal{A}$-linear,

$$
\nabla^{2}\left(v^{(k)} \rho\right)=\left(\nabla^{2} v^{(k)}\right) \rho, \quad v^{(k)} \in \mathcal{E}^{(k)}, \rho \in \Omega^{*} \mathcal{A}
$$

$\nabla^{2}$ is completely determinated by its restriction to $\mathcal{E}$. Such a restriction defines the curvature $\mathcal{F}(A)$ of the connection. One finds

$$
\mathcal{F}(A)(v \otimes a):=\nabla^{2}(v \otimes a)=(T(\alpha) v \otimes d A(\alpha)+T(\alpha) T(\beta) v \otimes A(\alpha) A(\beta)) a .
$$

We may therefore write

$$
\mathcal{F}(A)=T(\alpha) \otimes d A(\alpha)+T(\alpha) T(\beta) \otimes A(\alpha) A(\beta)
$$

Bianchi identity now reads

$$
[\nabla, \mathcal{F}(A)]=0
$$

This is really a trivial statement because left hand side is just $\nabla^{3}-\nabla^{3}$. One can explicitly check it by using expressions (5.15) and (5.19).

On $\mathcal{E}^{*}$, there is an $\Omega^{*} \mathcal{A}$-valued Hermitian structure given as follows. Any two $\mathcal{E}^{(k)}, \mathcal{E}^{\left(k^{\prime}\right)}$ are orthogonal if $k \neq k^{\prime}$, while

$$
<v_{1} \otimes \omega_{1}^{(k)}, v_{2} \otimes \omega_{2}^{(k)}>_{k}:=<v_{1}, v_{2}>\left(\omega_{1}^{(k)}\right)^{*} \omega_{2}^{(k)}:=\sum_{j=1}^{N} v_{1 j}^{*} v_{2 j}\left(\omega_{1}^{(k)}\right)^{*} \omega_{2}^{(k)}
$$

Now, we have

$$
\left(d \omega^{(k)}\right)^{*}=(-1)^{(k+1)} d \omega^{(k) *},
$$

so that compatibility of the connection with the Hermitian structure reads

$$
-<\nabla\left(v_{1} \otimes a\right), v_{2} \otimes b>+<v_{1} \otimes a, \nabla\left(v_{2} \otimes b\right)>=d<v_{1} \otimes a, v_{2} \otimes b>, a, b \in \mathcal{A} .
$$

By using (5.15) it reduces to

$$
-<T(\alpha) v_{1}, v_{2}>a^{*} A(\alpha)^{*} b+<v_{1}, T(\alpha) v_{2}>a^{*} A(\alpha) b=0 .
$$

If the $T(\alpha)$ 's are supposed to be hermitean, condition (5.24) requires that the $A(\alpha)$ 's are "real",

$$
A(\alpha)^{*}=A(\alpha)
$$

The continuum gauge group for $U_{N}$ now becomes its discretised version $\mathcal{U}_{N}$. It consists of $N \times N$ unitary matrices $g$ with $g_{i j} \in \mathcal{A}\left[13\right.$, 14, 15]. It acts on $\mathcal{E}^{(k)}$ according to $e^{(k)} \rightarrow g e^{(k)}$ and transforms the connection and curvature in the usual way:

$$
\nabla \rightarrow g \nabla g^{-1}, \quad \mathcal{F}(A) \rightarrow g \mathcal{F}(A) g^{-1}
$$


Here the matrices $g$ are understood to be tensored with the appropriate unit matrix. Furthermore, note that when dealing with representations, it is to be understood that functions like $g_{i j}$ are really of the form $\left[\begin{array}{cc}g_{i j} & 0 \\ 0 & g_{i j}\end{array}\right]$ so that $g$ is to be thought of as $\left[\begin{array}{ll}g & 0 \\ 0 & g\end{array}\right]$.

Consider $\mathcal{F}(A)^{\dagger}$ where $\dagger$ hermitean conjugates also $c_{i}$. $\mathcal{F}(A)^{\dagger}$ transforms in the same way as $\mathcal{F}(A)$. Hence the discrete Euclidean Yang-Mills action in dimension $d$ can be taken to be

$$
S_{Y M}(A)=\frac{1}{4 g^{2} a^{4-d}} \operatorname{Tr} \mathcal{F}(A)^{\dagger} \mathcal{F}(A),
$$

with $a$ being the lattice spacing and $g$ the coupling constant. This expression is gauge invariant. Here Tr indicates a trace over all indices including a Hilbert space trace for the creation-annihilation operators.

For a gauge group other than $\mathcal{U}_{N}$, we must suitably further restrict $g$ in the preceding discussion, and take $T(\alpha)$ to be the generators of this group.

It is remarkable that (5.27) is nothing but the Wilson action for a class of connections. [For an alternative derivation of Wilson action for gauge fields based on ideas of noncommutative geometry, see [29].]

Notice first that $F$ is a one-form and therefore can be used as gauge potential.

The gauge potential $-F$ is a fixed point of the gauge group which we now take to be $\mathcal{U}_{N}$ for specificity :

$$
g(-F) g^{-1}+g d g^{-1}=g(-F) g^{-1}+g\left[F, g^{-1}\right]=-F .
$$

So let

$$
A=-F+\Phi
$$

Then $\Phi$ transforms homogeneously:

$$
g: \Phi \rightarrow g \Phi g^{-1} .
$$

In one dimension, suppose we write

$$
\Phi=\left(\begin{array}{cc}
0 & u \Delta \\
\Delta^{\dagger} u^{\dagger} & 0
\end{array}\right) c, u \in \mathcal{U}_{N},
$$

This expression is compatible with the reality condition on $A$. Also since $\Delta g^{-1} \Delta^{\dagger} \in \mathcal{U}_{N}$, it is also consistent with (5.26), $u$ transforming according to

$$
u \rightarrow g u\left(\Delta g^{-1} \Delta^{\dagger}\right)
$$

This means that

$$
u(i) \rightarrow g(i) u(i) g(i+1)^{-1}
$$

where the argument is the site index. [We are shifting it from subscript to argument, as the former will be assigned to symmetry indices]. But this is exactly the transformation 
law of the Wilson link variable. Hence $u(i)$ can be identified with the link variable on link $z_{i, i+1}$ and $u(i)^{\dagger}$ with the one on $z_{i+1, i}$. Hereafter we will therefore often write

$$
u(i)=u_{i, i+1}
$$

with similar notations in higher dimensions.

Now consider $T^{2}$. For $T^{2}$, the ansatz for $\Phi$, compatible with "reality" and stable under gauge transformations is

$$
\begin{aligned}
\Phi & =\left[\mathcal{U}^{(1)} f_{+}^{(1)}+f_{+}^{(1)^{\dagger}} \mathcal{U}^{(1)^{\dagger}}\right] c_{1}+\left[\mathcal{U}^{(2)} f_{+}^{(2)}+f_{+}^{(2)^{\dagger}} \mathcal{U}^{(2)^{\dagger}}\right] c_{2} \\
& =\xi c_{1}+\eta c_{2}
\end{aligned}
$$

where $\mathcal{U}^{(k)}$ is really $\left(\begin{array}{cc}u^{(k)} & 0 \\ 0 & u^{(k)}\end{array}\right)$ and $\left(u^{(k) \dagger} u^{(k)}\right)_{i j}=\delta_{i j} \mathbf{1}, \mathbf{1}$ being the constant function in $\mathcal{A}$. The matrix $u^{(k)}(M)$ at site $M$ once more transforms as a link in the direction $k$. We must thus think of $u^{(k)}(M)$ as the Wilson variable on link from $M$ in the $k$-th direction.

Generalizations to all dimensions must now be obvious.

Having obtained the Wilson link in our way, we can evaluate the action. Note first that

$$
\mathcal{F}(A)=\Phi^{2}
$$

Hence

$$
S_{Y M}=\frac{1}{4 g^{2} a^{4-d}} \operatorname{Tr}\left(\Phi^{\dagger}\right)^{2} \Phi^{2}
$$

But this is just the Wilson action.

As an example, consider the two-dimensional torus $T^{2}$. For $T^{2}$,

$$
\Phi^{2}=(\xi \eta-\eta \xi) c_{1} c_{2}
$$

so that we have

$$
S_{Y M}=S_{Y M}^{(1)}+S_{Y M}^{(2)}
$$

where

$$
\begin{gathered}
S_{Y M}^{(1)}=-\frac{1}{2 g^{2} a^{2}} \operatorname{Tr} \xi \eta \xi \eta, \\
S_{Y M}^{(2)}=\frac{1}{2 g^{2} a^{2}} \operatorname{Tr} \xi^{2} \eta^{2} .
\end{gathered}
$$

For each $(\alpha, \beta)$ in the notation of (4.8), $S_{Y M}^{(1)}$ has a term

$$
S_{Y M}^{(1)}(\alpha \beta)=-\frac{1}{g^{2} a^{2}} \operatorname{Tr}\left\{\Lambda_{c l}(\alpha, \beta)+\Lambda_{a c l}(\alpha, \beta)\right\}
$$

where $\Lambda_{c l}(\alpha, \beta)$ is the product of the Wilson links around the plaquette in the clockwise direction starting at $(\alpha, \beta)$, and similarly $\Lambda_{a c l}(\alpha, \beta)$ for the anticlockwise direction. A similar calculation gives

$$
S_{Y M}^{(2)}(\alpha \beta)=\frac{2}{g^{2} a^{2}} \operatorname{Tr} \mathbb{I}
$$


The contribution to the action of each $(\alpha, \beta)$ is therefore

$$
S_{Y M}(\alpha \beta)=\frac{2}{g^{2} a^{2}} \operatorname{Tr}\left\{\mathbb{I}-\frac{1}{2}\left(\Lambda_{c l}(\alpha, \beta)+\Lambda_{a c l}(\alpha, \beta)\right)\right\} .
$$

$S_{Y M}$ is the sum over $\alpha, \beta$ of these terms and their hermitean conjugates and is just the Wilson action.

This calculation for $T^{2}$ readily generalizes to higher dimensions.

\section{The Dirac Action}

Let us consider a spacetime of dimension $K$. If $M$ is the desired internal dimension of the Dirac field, introduce

$$
\widetilde{\mathcal{E}}=\mathcal{A} \otimes \mathbb{C}^{M}:=\mathcal{A}^{M}=\left\{e=\left(e_{1}, e_{2}, \cdots e_{M}\right) ; e_{i} \in \mathcal{A}\right\}
$$

Let us also consider

$$
\tau_{1}=\left(\begin{array}{ll}
0 & 1 \\
1 & 0
\end{array}\right)
$$

acting on $\Omega^{*} \mathcal{A}$ and

$$
T_{1}=\tau_{1} \otimes 1
$$

acting on $\widetilde{\mathcal{E}}$.

Note that $T_{1}$ is gauge invariant :

$$
\left(\begin{array}{cc}
g & 0 \\
0 & g
\end{array}\right) T_{1}\left(\begin{array}{cc}
g^{\dagger} & 0 \\
0 & g^{\dagger}
\end{array}\right)=T_{1} .
$$

The connection $\nabla$ acts on an element $e \in \widetilde{\mathcal{E}}$ according to

$$
\nabla e=\left[F+A, T_{1}\right] e .
$$

Let us consider the connection $\nabla$ of the form (5.31) and (5.35) generalised to $K$ dimensions. The component form of (6.5) then is

$$
(\nabla e)_{i}=\left[\Phi, T_{1}\right]_{i j} e_{j}, \quad 1 \leq i, j \leq M,
$$

$i, j$ being internal indices.

We can clarify the meaning of (6.5) further by examining the $K=1$ case. Then in the notation where gauge transformation has the doubled form $\left(\begin{array}{ll}g & 0 \\ 0 & g\end{array}\right)$, the right hand side of (6.6) reads

$$
\left(\begin{array}{cc}
\left(u_{i j} \Delta-\Delta^{\dagger} u_{i j}^{\dagger}\right) e_{j} & 0 \\
0 & -\left(u_{i j} \Delta-\Delta^{\dagger} u_{i j}^{\dagger}\right) e_{j}
\end{array}\right) c
$$


so that when $u=\mathbb{I}$, the upper and lower diagonals consist of forward and backward finite differences.

We next introduce the $\gamma$-matrices. Let $V$ be the vector space carrying the representation of $\gamma$ 's [30]. Let

$$
\mathcal{E}_{D}=\widetilde{\mathcal{E}} \otimes_{\mathbb{C}} V .
$$

An element of $\mathcal{E}_{D}$ is a "spinor" $\psi$ with components $\psi_{\lambda}^{\alpha} \in \mathcal{A}(\alpha=1,2, \cdots M ; \lambda=1,2, \cdots)$.

There are $K \Phi^{(i)}$ 's corresponding to differentiations in $K$ directions. Therefore we can write

$$
\Phi=\sum \Phi^{(k)},
$$

$\Phi^{(k)}$ being proportional to $c_{k}$ in the $K$-dimensional version of (5.35).We can hence write

$$
(\nabla e)_{i}=\sum_{k}\left[\Phi^{(k)}, T_{1}\right]_{i j} e_{j} c_{k} \equiv \sum D_{i j}^{(k)} e_{j} c_{k} .
$$

There being also $K \gamma$-matrices $\gamma_{k}$, we can form the Dirac operator

$$
\begin{aligned}
& D=\gamma_{k} D^{(k)}+m, \\
& m=\text { Mass term },
\end{aligned}
$$

and the Dirac equation

$$
[D \psi]_{\lambda}^{\alpha} \equiv\left(\gamma_{k}\right)_{\lambda}^{\rho} D_{\alpha \beta}^{(k)} \psi_{\rho}^{\beta}+m \psi_{\lambda}^{\alpha}=0 .
$$

[There is no distinction between upper and lower internal indices, the internal metric being the Kronecker delta.]

It is easy to check that (6.12) gives Wilson's formulation [2] for Dirac equation in a doubled form. The source of the doubling is illustrated by the appearance of both forward and backward differences in (6.7) for $u=\mathbb{I}$. We get exactly Wilson's discretisation by retaining only one of these equations.

We thus have a geometrical justification of the Wilson actions for gluons and fermions which emerge naturally from the connection and curvature of a gauge theory defined on a noncommutative lattice. It is however disappointing that this approach is yet to suggest a solution for the doubling problem of chiral fermions.

\section{Topological Action in 2d}

There is an analogue of the QCD $\theta$ term in the continuum two-dimensional QED. If $d A$ is the curvature, it is $\theta \int d A$. Being independent of the metric, it is topological. It also integrates to an integral of the connection, just as the $\theta$-term integrates to an integral of the Chern-Simons term. The algebraic formulation and noncommutative geometry have natural candidates for the discrete analogue of this term, often called "topological susceptibility". We now describe these discretisations. 


\subsection{The First Choice}

The discretised curvature in this choice is $\mathcal{F}(A)=d A+A^{2}=\Phi^{2}$ [the $A^{2}$-term being present even for $U(1)$ gauge group.]. Hence the 2 d lattice topological term is

$$
S_{\theta}=\theta \int\left(d A+A^{2}\right)+\text { h.c. }=\theta \operatorname{Tr} \Phi^{2} F_{+}^{(1) \dagger} F_{+}^{(2) \dagger}+\text { h.c. }
$$

When $A$ is a pure gauge, $\mathcal{F}(A)=0$ and $S_{\theta}$ vanishes, as it should.

This proposal for topological susceptibility has a certain merit. It flows from the formalism without strain or doctoring, and also has the correct (naive) continuum limit.

But it is defective as well for the following reasons:

i) It is not gauge invariant. Thus since $\Phi \rightarrow g \Phi g^{-1}$ under a gauge transformation $g$,

$$
\operatorname{Tr} \Phi^{2} F^{(1) \dagger} F^{(2) \dagger} \rightarrow \operatorname{Tr}\left\{\Phi^{2}\left[g^{-1} F_{+}^{(1) \dagger} F_{+}^{(2) \dagger} g\right]\right\}
$$

and $F_{+}^{(1) \dagger} F_{+}^{(2) \dagger}$ need not commute with $g$. The root of this difficulty is lack of graded commutativity in $\Omega^{*} \mathcal{A}$.

ii) It does not integrate to an integral of $A$.

Both these problems occur in $4 \mathrm{~d}$ too. But it is possible to manage them in a reasonable manner in either dimension as we shall now see in $2 \mathrm{~d}$.

In $2 \mathrm{~d}$, as regards $\mathrm{i}$ ), let us restrict gauge transformations by requiring that

$$
g^{-1} F_{+}^{(1) \dagger} F_{+}^{(2) \dagger} g=F_{+}^{(1) \dagger} F_{+}^{(2) \dagger} .
$$

In the plaquette of Fig. 3, this means that the gauge transformations are constrained to be the same at $(\alpha, \beta)$ and $(\alpha+1, \beta+1)$. Thus in a square lattice with $N^{2}$ points, we can freely choose the value of $g$ at the $N$ points along say the bottom row. This may not be too bad, this constraint superficially comparing favorably with the $1 / N$ approximation [31].

The second limitation above can be overcome if

$$
u^{(2)} F_{+}^{(1) \dagger} F_{+}^{(2) \dagger} u^{(2) \dagger}=F_{+}^{(1) \dagger} F_{+}^{(2) \dagger}
$$

or

$$
\begin{gathered}
W^{+}(\alpha, \beta)=W^{-}(\alpha, \beta-1) \\
W^{-}(\alpha, \beta):=\operatorname{tr}\left[u_{a d}^{(2)} u_{d c}^{(1)}\right], \quad W^{+}(\alpha, \beta):=\operatorname{tr}\left[u_{a b}^{(1)} u_{b c}^{(2)}\right]
\end{gathered}
$$

where for notational simplicity, we have relabeled the points $(\alpha, \beta),(\alpha+1, \beta),(\alpha+1, \beta+$ $1),(\alpha, \beta+1)$ as $a, b, c, d$. Also we are using the notation (5.34) so that, for example, 


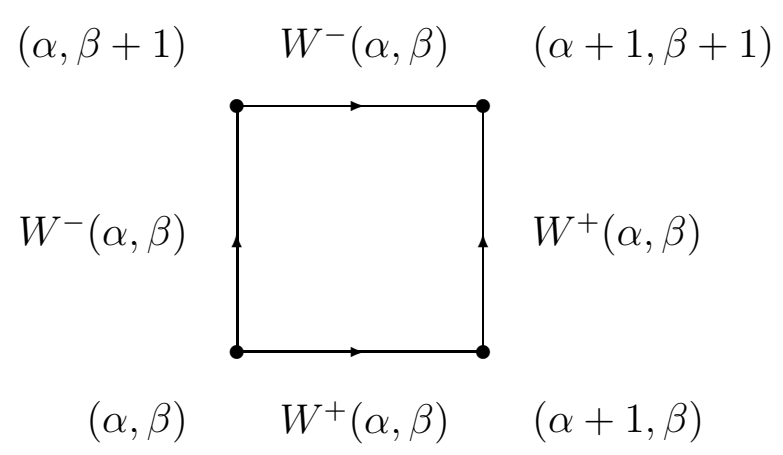

Fig. 3. Gauge invariance requires that gauge transformations are the same at $(\alpha, \beta)$ and $(\alpha+1, \beta+1)$. $W^{+}(\alpha, \beta)$ and $W^{-}(\alpha, \beta)$ are the expressions in (7.5).

$u^{(2)}[(\alpha \beta)]=u_{(\alpha, \beta)(\alpha, \beta+1)}^{(2)}=u_{a d}^{(2)}$. Note further that $\operatorname{tr}$ (with lower case tr) is trace over internal indices. Although redundant in the present $U(1)$ case, we leave it in here for uniformity. (It will be removed in Section 7.2.)

This constraint is similar to the condition on $g$ above. It is fulfilled if $u^{(2)}$ is invariant by translation from $(\alpha, \beta)$ to $(\alpha+1, \beta+1)$. It can be regarded as a gauge fixing condition, the constrained gauge transformations (7.3) identifying the left-over gauge group after the partial gauge fixing (7.4.7.5). We will expand on this further in Section 8.

We have yet to show that $S_{\theta}$ integrates to a surface term with (7.4, 7.5). Now

$$
S_{\theta}=\theta \sum_{(\alpha, \beta)}\left[W^{-}(\alpha, \beta)-W^{+}(\alpha, \beta)\right]
$$

If $a^{\prime}[=(\alpha, \beta-1)]$ is the point vertically below $a$, then

$$
W^{+}(a)=W^{-}\left(a^{\prime}\right)
$$

by (7.5), and $W^{-}\left(a^{\prime}\right)$ cancels $W^{+}(a)$ in the sum in (7.6).

Suppose we are integrating over the rectangular lattice of Fig. 4. It follows from these cancellations that $S_{\theta}$ is a surface term:

$$
S_{\theta}=\theta \sum_{i}\left[W^{-}\left(a_{i}^{\prime}\right)-W^{+}\left(b_{i}^{\prime}\right)\right]
$$

\subsection{Alternatives}

The boundary terms (7.7) suggest an alternative topological term which has the virtue that it can dispense with the gauge fixing condition (7.5). But the constraint (7.3) on the gauge group is still needed. 

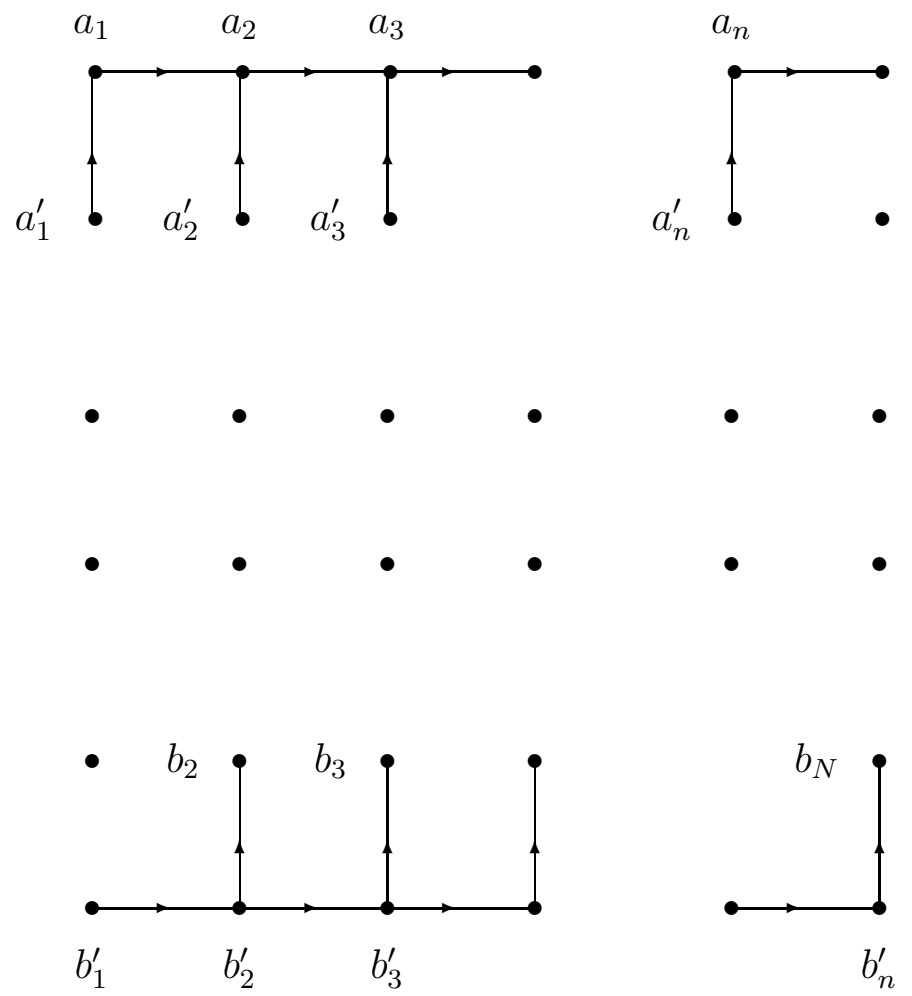

Fig. 4. Here is shown the rectangular lattice of integration. Only the surface terms indicated by arrows remain after the gauge fixing condition (7.5).

The idea for this alternative follows from (7.7) and the continuum results. We first write $\sum_{i} W^{-}\left(a_{i}^{\prime}\right)$ for example as a one-dimensional integral:

$$
\sum_{i} W^{-}\left(a_{i}^{\prime}\right)=\theta \sum_{a_{i}}\left\langle a_{i}\left|\left\{\left(f_{+}^{(2)^{\dagger}} u^{(2)} f_{+}^{(2)}\right)\left(u^{(1)} f_{+}^{(1)}\right)\right\} f_{+}^{(1)^{\dagger}}\right| a_{i}\right\rangle .
$$

This is just the integral of the one-form

$$
\mathcal{A}=\left(f_{+}^{(2)^{\dagger}}\right) u^{(2)}\left(f_{+}^{(2)}\right)\left(u^{(1)} f_{+}^{(1)}\right) c_{1}
$$

over the top horizontal line. It is to be compared with the integral at the final time of the connection $A$ in the continuum theory.

Now the topological term in the latter is $\theta \int d A$. In analogy, we can write its discrete analogue as follows:

$$
S_{\theta}^{\prime}=\theta \int d \mathcal{A} .
$$

Now with the constraint (7.3) on $g, \mathcal{A}$ transforms homogeneously and not as a connection under gauge transformations. For this (and other) reasons, $d \mathcal{A}$ is not gauge invariant. 
Still $S_{\theta}^{\prime}$ is, as it integrates to gauge invariant boundary terms. This is so without any condition on $u^{(j)}$. If we choose to impose the rule (7.4) on $u^{(2)}$, then $S_{\theta}^{\prime}$ becomes $S_{\theta}$.

We can see this by just writing out $S_{\theta}^{\prime}$ :

$$
\begin{aligned}
S_{\theta}^{\prime}= & \theta \sum_{(\alpha, \beta)}\langle(\alpha, \beta)| f_{+}^{(2)}\left(f_{+}^{(2)^{\dagger}} u^{(2)} f_{+}^{(2)} u^{(1)} f_{+}^{(1)}\right) f_{+}^{(1)^{\dagger}} f_{+}^{(2)^{\dagger}-} \\
& -\left(f_{+}^{(2)^{\dagger}} u^{(2)} f_{+}^{(2)} u^{(1)} f_{+}^{(2)}\right) f_{+}^{(2)^{\dagger}} f_{+}^{(1)} f_{+}^{(1)^{\dagger}}|(\alpha, \beta)\rangle
\end{aligned}
$$

where the sum is over an appropriate range and the traces over creation-annihilation operators have been performed. The first sum here gives the $W^{-}(\alpha, \beta)$ terms. On putting in $u^{(2)}(\alpha, \beta)=u^{(2)}(\alpha+1, \beta+1)$, the second sum gives the $W^{+}(\alpha, \beta)$ terms.

We can also begin from $W^{+}\left(b_{i}^{\prime}\right)$ to find another candidate $S_{\theta}^{\prime \prime}$ for topological susceptibility, giving us three possibilities in all for this term. They are all different if $u^{(2)}$ is not constrained, but become the same with a constrained $u^{(2)}$. Only future practice can decide the best choice among them or their combinations.

\section{Topological Action in Four Dimensions}

We now turn our attention to the QCD topological term. In this case, the group being $S U(3)_{c}, \mathcal{E}$ is $\mathcal{A}^{3}$ and $u^{(i)}$ are both unitary and unimodular.

\subsection{The First Choice}

There is as before an effortless construction of a discrete "topological" action $\mathcal{S}_{\theta}$ here as well. If $\mathcal{F}(A)$ is the curvature, it is

$$
\begin{gathered}
\mathcal{S}_{\theta}=\frac{\theta}{8 \pi^{2}} \int \operatorname{tr} \mathcal{F}(A)^{2}:=\frac{\theta}{8 \pi^{2}} \operatorname{Tr} \mathcal{F}(A)^{2} \Pi_{i} f_{+}^{(i) \dagger} c_{i}^{\dagger}, \\
\Pi_{i} f_{+}^{(i) \dagger} c_{i}^{\dagger}=f_{+}^{(1) \dagger} f_{+}^{(2) \dagger} f_{+}^{(3) \dagger} f_{+}^{(4) \dagger} c_{1}^{\dagger} c_{2}^{\dagger} c_{3}^{\dagger} c_{4}^{\dagger} .
\end{gathered}
$$

When $A$ is a pure gauge, $\mathcal{F}(A)=0$ and $\mathcal{S}_{\theta}$ vanishes, as it should.

It can be shown that the naive continuum limit of $\mathcal{S}_{\theta}$ is the continuum $\theta$-term.

Gauge invariance of $\mathcal{S}_{\theta}$ requires a constraint like (7.3) on gauge transformations $g$, namely,

$$
g\left(\Pi_{i} f_{+}^{(i) \dagger} c_{i}^{\dagger}\right)=\left(\Pi_{i} f_{+}^{(i) \dagger} c_{i}^{\dagger}\right) g .
$$

If the points of the $T^{4}$-lattice are labeled by $(\alpha, \beta, \gamma, \delta)$, (8.2) means that

$$
g(\alpha+1, \beta+1, \gamma+1, \delta+1)=g(\alpha, \beta, \gamma, \delta)
$$


Thus the value of $g$ along the diagonal is constrained to be a constant. In other words, if the number of points along each line of the lattice is $N$ so that the total number of points is $N^{4}$, we can freely choose $N^{3}$ out of $N^{4}$ values of $g$, the remaining ones being fixed by (8.3). Naively this appears an acceptable limitation.

Just as the $2 \mathrm{~d}$ topological term, the QCD $\theta$-term in the continuum integrates to a surface term whereas like its two dimensional partner, $\mathcal{S}_{\theta}$ does not do so without a gauge fixing condition. But before stating this condition, let us write out $\mathcal{S}_{\theta}$.

We define the hypercube $C(\alpha, \beta, \gamma, \delta)$ attached to the vertex $(\alpha, \beta, \gamma, \delta)$ as the cube with $(\alpha, \beta, \gamma, \delta)$ and $(\alpha+1, \beta+1, \gamma+1, \delta+1)$ as diagonally opposite vertices. Its vertices are $(\alpha+i, \beta+j, \gamma+k, \delta+l)$ where $i, j, k, l \in\{0,1\}$. There are $4 !=24$ terms in $\mathcal{S}_{\theta}$ from each cube $C(\alpha, \beta, \gamma, \delta)$ corresponding to that many permutations of $1,2,3,4$. Given one such permutation or order, say $3,1,2,4$, we can pick a set of ordered vertices of the cube, those in this example being $\xi_{0}=(\alpha, \beta, \gamma, \delta), \xi_{1}=(\alpha, \beta, \gamma+1, \delta), \xi_{2}=(\alpha+1, \beta, \gamma+$ $1, \delta), \xi_{3}=(\alpha+1, \beta+1, \gamma+1, \delta), \xi_{4}=(\alpha+1, \beta+1, \gamma+1, \delta+1)$. Thus for each permutation $P$, there are five ordered vertices from $(\alpha, \beta, \gamma, \delta)$ to the diagonally opposite corner $(\alpha+1, \beta+1, \gamma+1, \delta+1)$. Now join the successive vertices to get four links and take the trace of the product of the Wilson variables along these links. Multiplying this expression by $\frac{\theta}{8 \pi^{2}}(-1)^{\epsilon(P)}$, where $\epsilon(P)$ is the signature of $P$, we get the contribution of $P$ to $\mathcal{S}_{\theta}$. In the example above, this contribution is

$$
\frac{\theta}{8 \pi^{2}} \operatorname{Tr} u_{\xi_{0} \xi_{1}}^{(3)} u_{\xi_{1} \xi_{2}}^{(1)} u_{\xi_{2} \xi_{3}}^{(2)} u_{\xi_{3} \xi_{4}}^{(4)}
$$

We can concisely write a typical term as $\frac{\theta}{8 \pi^{2}}(-1)^{\varepsilon(P)} W_{P(1234)}(\alpha \beta \gamma \delta)$, (8.4) being $\frac{\theta}{8 \pi^{2}} W_{3124}(\alpha \beta \gamma \delta)$. The total contribution $\frac{\theta}{8 \pi^{2}} W(\alpha \beta \gamma \delta)$ from $C(\alpha \beta \gamma \delta)$ is its sum over $P$,

$$
\frac{\theta}{8 \pi^{2}} W(\alpha \beta \gamma \delta)=\frac{\theta}{8 \pi^{2}} \sum_{P}(-1)^{\varepsilon(P)} W_{P(1234)}(\alpha \beta \gamma \delta)
$$

while

$$
\mathcal{S}_{\theta}=\frac{\theta}{8 \pi^{2}} \sum_{(\alpha \beta \gamma \delta)} W(\alpha \beta \gamma \delta)
$$

This expression seems a great deal simpler than existing proposals for topological susceptibility which typically contain products of eight $u^{(i)}$ 's per hypercube [16, 17, 18] while there are only 4 here.

We have already addressed the question of gauge invariance of $\mathcal{S}_{\theta}$. It remains to discuss the analogue of item ii) in $2 d$. Just as in $2 d$, the continuum integral of $\operatorname{tr} F \wedge F$ integrates to a surface integral. But $\mathcal{S}_{\theta}$ does so only with a gauge condition, again like in $2 d$, as we now explain.

We can write

$$
\begin{aligned}
W(\alpha \beta \gamma \delta) & =W^{+}(\alpha \beta \gamma \delta)-W^{-}(\alpha \beta \gamma \delta) \\
W^{ \pm}(\alpha \beta \gamma \delta) & =\sum_{\varepsilon(P)= \pm 1} W_{P(1234)}(\alpha \beta \gamma \delta) .
\end{aligned}
$$


The analogue of the $2 d$ gauge condition now is

$$
W^{+}(\alpha \beta \gamma \delta)=W^{-}(\alpha \beta \gamma(\delta-1)) .
$$

It cannot be written in a form like (7.4) because of the noncommutativity of the nonabelian $u^{(i)}$ 's.

It should be evident that $\mathcal{S}_{\theta}$ integrates to a surface term with (8.9). If the $\delta$-direction is thought of as time direction, and $\delta \in\{1,2, \ldots, N\}$, then $\mathcal{S}_{\theta}$ consists of integrals at future and past time boundaries:

$$
\mathcal{S}_{\theta}=\frac{\theta}{8 \pi^{2}} \sum_{(\alpha \beta \gamma)}\left\{W^{+}(\alpha \beta \gamma 1)-W^{-}(\alpha \beta \gamma(N-1))\right\} .
$$

In four dimensions, the analogue of $W(\alpha \beta \gamma \delta)$ consists of sums of terms, a typical one being like (8.4), with $u_{\eta \xi}^{(j)}$ interpreted now as $\mathrm{P} \exp \int_{\xi}^{\eta} A$.

The expression (8.10) can be further expanded in lattice spacing, the first nontrivial term then involves the connection. In a similar manner the first nontrivial term in $W(\alpha \beta \gamma \delta)$ involves the Chern-Simons 3-form. The gauge condition (8.9) in the continuum corresponds to a constraint on this form.

\section{The Chern-Simons Term}

In the continuum the Chern-Simons (CS) term exists in all odd dimensions, and is the integral of the CS form [19]. It has played a crucial role in many recent studies in theoretical physics. There have also been previous proposals for its discrete analogue [20, 21].

In this section we briefly show that there are candidates for this term in the algebraic approach. For brevity we will limit ourselves to 1 and 3d. The candidates we will propose fail to have several of the properties of the continuum Chern-Simons in an exact manner, fulfilling them only up to corrections vanishing in the naive continuum limit.

One candidate in the discrete for this term is obtained by integrating the discretized form of CS density. It has the expression

$$
\frac{k}{8 \pi^{2}} \int \operatorname{tr}\left(A d A+\frac{2}{3} A^{3}\right)=\frac{k}{8 \pi^{2}} \operatorname{Tr}\left(A d A+\frac{2}{3} A^{3}\right) f_{+}^{(1) \dagger} f_{+}^{(2) \dagger} f_{+}^{(3) \dagger} c_{1}^{\dagger} c_{2}^{\dagger} c_{3}^{\dagger}
$$

for any gauge group, where $k$ is a normalization constant.

Lack of graded commutativity means that $A d A \neq d A A$. For this reason, it seems best to symmetrize $A d A$ and write for a discrete CS term,

$$
\begin{aligned}
\mathcal{A}_{C S} & =\frac{k}{8 \pi^{2}} \operatorname{Tr}\left(\frac{1}{2}(A d A+d A A)+\frac{2}{3} A^{3}\right) f_{+}^{(1)^{\dagger}} f_{+}^{(2)^{\dagger}} f_{+}^{(3)^{\dagger}} c_{1}^{\dagger} c_{2}^{\dagger} c_{3}^{\dagger} \\
& =\frac{k}{8 \pi^{2}} \operatorname{Tr}\left(\frac{1}{2}(A \mathcal{F}(A)+\mathcal{F}(A) A)-\frac{1}{3} A^{3}\right) f_{+}^{(1)^{\dagger}} f_{+}^{(2)^{\dagger}} f_{+}^{(3)} c_{1}^{\dagger} c_{2}^{\dagger} c_{3}^{\dagger} .
\end{aligned}
$$


Substituting $A=-F+\Phi$ and $\mathcal{F}(A)=\Phi^{2}$, it becomes

$$
\mathcal{A}_{C S}=\frac{k}{8 \pi^{2}} \operatorname{Tr}\left(\frac{2}{3} \Phi^{3}-\frac{1}{6}\left(F \Phi^{2}+\Phi^{2} F\right)+\frac{1}{3}(\Phi F \Phi-F \Phi F)\right) f_{+}^{(1)^{\dagger}} f_{+}^{(2)^{\dagger}} f_{+}^{(3)}{ }^{\dagger} c_{1}^{\dagger} c_{2}^{\dagger} c_{3}^{\dagger} .
$$

\section{The Hamiltonian Formulation}

In the Hamiltonian formulation, the poset is the topological lattice of the spatial manifold. The constructions of the preceding sections, and especially of Section 5, are still largely valid if physical interpretations are suitably modified and names are changed accordingly. In particular, $d A+A^{2}$ is to be identified with the magnetic field $B(A)$,

$$
B(A)=d A+A^{2}:=\mathcal{F}(A)
$$

and $S_{Y M}(A)$ with the "potential energy" term (proportional to) $V(A)$ of the Yang-Mills Hamiltonian:

$$
V(A)=\operatorname{Tr} B(A)^{\dagger} B(A)
$$

It remains to define the electric field, the Gauss law constraint and finally the Hamiltonian. We take advantage of earlier work of Rajeev [22] in formulating these definitions.

Let us introduce an orthonormal basis $\phi_{n}$ for the Lie algebra-valued one-forms, as it will be useful shortly:

$$
\begin{aligned}
\left(\phi_{n}, \phi_{m}\right) & =\operatorname{Tr} \phi_{n}^{\dagger} \phi_{m}=\delta_{n m}, \\
\phi_{n} & =\phi_{n}^{\alpha} T(\alpha), \quad \phi_{n}^{\alpha} \in \Omega^{1} \mathcal{A}, \quad\left(\phi_{n}^{\alpha}\right)^{*}=\phi_{n}^{\alpha} .
\end{aligned}
$$

We will work in the Schrödinger representation where wave functions are $\mathbb{C}$-valued functions of $A$.

In the continuum, the electric field $E=\left(E_{1}, E_{2}, \cdots, E_{M}\right), M=K-1, E_{i}=E_{i}^{\alpha} T(\alpha)$, consists of conjugate operators to $A_{j}=A_{j}^{\alpha} T(\alpha)$ [M being the spatial dimension]. If $\chi$ is a functional of $A$, then, at a formal level, $i E_{i}^{\alpha}(x)$ is the operator $\frac{\delta}{\delta A_{i}^{\alpha}(x)}, x$ being a spatial point. Hence the test function space for $E$ consists of Lie algebra-valued one forms, $f=\left(f_{1}, f_{2}, \cdots, f_{M}\right), f_{i}=f_{i}^{\alpha} T(\alpha),\left(f_{i}^{\alpha}\right)^{*}=f_{i}^{\alpha}$, the pairing $\langle f, E\rangle$ acting on $\chi$ according to 22

$$
(i\langle f, E\rangle \chi)(A)=\lim _{t \rightarrow 0} \frac{\chi(A+t f)-\chi(A)}{t}, \quad t \in \mathbb{R} \text {. }
$$

In a similar manner, for a poset, we introduce the pairing $\langle\lambda, E\rangle$ for $\lambda \in \underline{\mathcal{U}_{N}} \otimes\left(\Omega^{1} \mathcal{A}\right)$, where $\underline{\mathcal{U}}_{N}$ is the Lie algebra of $\mathcal{U}_{N}$. Thus $\lambda$ is a Lie algebra valued one-form, $\lambda \overline{=\lambda}(\alpha) T(\alpha)$. We also require that

$$
\lambda(\alpha)^{*}=\lambda(\alpha)
$$


just as $A(\alpha)^{*}=A(\alpha)$.

$\langle\lambda, E\rangle$ acts on wave functions according to

$$
(i\langle\lambda, E\rangle \psi)(A)=\lim _{t \rightarrow 0} \frac{\psi(A+t \lambda)-\psi(A)}{t} .
$$

There is also an elegant statement of Gauss' law in this formulation. Let

$$
\Lambda \in \underline{\mathcal{U}_{N}} \otimes \mathcal{A}
$$

so that

$$
\Lambda=\Lambda(\alpha) T(\alpha), \quad \Lambda(\alpha) \in \mathcal{A}
$$

$\Lambda$ is a Lie algebra-valued function. Let us also require that

$$
\Lambda(\alpha)^{*}=\Lambda(\alpha)
$$

The covariant derivative of $\Lambda$ is

$$
\nabla_{A} \Lambda \equiv\left[F, \Lambda^{\alpha}\right] T(\alpha)+A^{\beta} \Lambda^{\alpha}[T(\beta), T(\alpha)] .
$$

The Gauss' law is just the condition

$$
\left\langle\nabla_{A} \Lambda, E\right\rangle \psi=0
$$

on the physical states $\psi$.

The Yang-Mills Hamiltonian is the operator

$$
H=\frac{1}{2 e^{2}}\left[\sum_{n}\left(\phi_{n}, E\right)^{\dagger}\left(\phi_{n}, E\right)+\hat{V}\right]
$$

where

$$
(\hat{V} \psi)(A)=V(A) \psi(A)
$$

and the first term in $H$ is to be defined using (10.6) and the scalar product below.

Finally, the scalar product on wave functions is

$$
(\psi, \chi)=\int\left(\Pi_{\alpha} d A^{\alpha}\right) \psi^{*}(A) \chi(A) .
$$

The Hamiltonian gauge theory on topological lattices is defined by this scalar product, the Gauss' law (10.11) and the Hamiltonian (10.12). It describes a bunch of oscillators with the exotic restriction (10.11) on wave functions.

There is one more result we wish to discuss here, namely the use of the CS functional in the Hamiltonian context. As is well known, with its help, we can readily change the $\theta$-angle associated with a state vector $\psi$ for $M=3$ [Cf. Jackiw in [19]]. 
Thus let us suppose that a wave functional $\psi_{0}$ in the continuum is invariant under all gauge transformations, including those not vanishing at infinity. Then $\psi_{0}$, being invariant under all gauge transformations, corresponds to the continuum gauge theory with zero $\theta$-angle. The wave functional $\psi_{\theta}$ in the continuum gauge theory with non-zero $\theta$ is given by

$$
\psi_{\theta}(A)=[\exp i \theta C(A)] \psi_{0}(A) .
$$

$C(A)$ being the continuum CS term.

In an analogous manner we can try using a CS term of Section 9 to change the $\theta$ angle of a quantum state in the discrete context. We have not however explored this possibility in any depth.

The discussion of this Section can be straightforwardly adapted also to groups such as $S U_{N}$.

\section{Final Remarks}

In this paper we have made progress on two fronts in formulating quantum physics on lattices. The first front is conceptual and concerns methods to approximate topology as well in the discretization of continua. In our approach to this important issue, we have adopted Sorkin's ideas [6, 25] to topologize cubical and simplicial decompositions of manifolds. We have then argued that the noncommutative algebra of cochains under the cup product fully captures the above topological data. In addition it is also perfectly adapted for quantum physics when combined with the Connes-Lott technology for noncommutative geometry [13, 14, 15].

In this manner we have developed lattice gauge theories for cubical lattices and shown the natural emergence of Wilson's action therefrom for gauge and spin half fields.

The second front where this paper shows progress concerns topological actions like the QCD $\theta$ term and the Chern-Simons action. Our proposals for discretizations have several apparently superior features to alternative existing models.

There is one aspect regarding these topological terms which bears emphasis. It seems impossible to formulate their discrete analogue without losing, at least in an approximate way, one or another of their basic properties. The root of this difficulty is the well-known impossibility of converting cochains into an associative, graded commutative algebra. This could be an indication that their reguralization in continuum interacting field theories leads to anomalies. The latter may be intolerable, for example they may disturb gauge invariance. If so, that would be a good reason to choose $\theta=0$ for example in QCD, thereby also resolving the strong CP problem [32].

In this paper we have emphasized the action functional formulation. It is what is needed for functional integral quantization. Despite its superiority for numerical work 
over the Hamiltonian, the latter as well has its place in discrete physics. Therefore we have also devoted a section for its treatment.

The substantial portion of this paper concentrates on cubical decompositions of the manifold. Preliminary explorations of the algebraic approach for simplicial decompositions have also been made with Allen Stern [33]. A notable surprise we have encountered in this work is that it is not in general possible to realize the differential $d$ as a graded commutator with an operator like $F_{+}$or $F$. We hope to report on this result elsewhere.

The effectiveness of our topological approach to discrete quantum physics and its corresponding algebraic description is not limited to gauge theories. It is very useful for example for soliton physics, and preserves fragile but important features like winding numbers, ruined by ordinary discretizations. This point has been already explained elsewhere [3] and will be thoroughly developed in a forthcoming work.

There is one other setting in which these methods lead to interesting results. Preliminary work indicates that our discrete quantum physics may be effective for studying topology change and also for formulating discrete topological quantum field theories. We plan to report on this research after it meets a measure of success.

\section{Acknowledgments}

We acknowledge with gratitude the collaboration of Elisa Ercolessi, Arshad Momen and Gianni Sparano during the early stages of this work, and our fruitful discussions with them, Beppe Marmo, Peter Michor, Apoorva Patel and Al Stern about its diverse aspects. In particular the relevance of cup algebra to our research was brought to our attention by Peter. Much of this work was carried out during our stay at the Erwin Schrödinger Institute for Mathematical Physics (ESI) in Vienna. We also thank ESI for financial support, and Beppe and Peter for making our stay in Vienna a rewarding experience. This work was in addition supported by the Istituto Italiano di Studi Filosofici by the Department of Energy U.S.A. under contract numbers DE-FG-02-84ER40173 and, DEFG02-85ER40231, and by INFN (Italy). 


\section{References}

[1] J. Donoghue, E. Golowich and B. Holstein, Dynamics of the Standard Model (Cambridge University Press, London, 1992).

[2] I. Montvay and G. Münster , Field Theories on the Lattice (Cambridge University Press, London, 1994).

[3] A.P. Balachandran, G. Bimonte, E. Ercolessi and P. Teotonio-Sobrinho, Nucl. Phys. B 418 (1994) 477.

[4] A.P. Balachandran, G. Bimonte, E. Ercolessi, G. Landi, F. Lizzi, G. Sparano and P. Teotonio-Sobrinho, Nucl. Phys. B (Proc. Suppl.) 37C (1995) 20 (hepth/9403067).

[5] A.P. Balachandran, G. Bimonte, E. Ercolessi, G. Landi, F. Lizzi, G. Sparano and P. Teotonio-Sobrinho, J. Geom. Phys. 18 (1996) 163 and hep-th/9510217.

[6] R.D. Sorkin, Int. J. Theor. Phys. 30 (1991) 323.

[7] For related work focusing attention on topology and discretizations, see J. Kijowski, Reports Math. Phys. 11 (1977) 97; A.V. Evako, Syracuse University preprint, grqc/9402035; A.A. Grib and R.R. Zapatrin, gr-qc/9507033 and ref. [8]

[8] P. Teotonio-Sobrinho, Nucl. Phys. B452 (1995) 526 and hep-th/9505028.

[9] J.R. Munkres, Elements of Algebraic Topology (Addison-Wesley Pub. Comp. 1984).

[10] E. Seiler, I. O. Stamatescu and D. Zwazinger, Nucl. Phys. B239 (1984) 177 and 204.

[11] D. Birmingham and P. Rakowski, Phys. Lett. B299 (1993) 299.

[12] J.M.G. Fell and R.S. Doran, Representations of*-Algebras, Locally Compact Groups and Banach *-Algebraic Bundles [Academic Press, 1988].

[13] A. Connes and J. Lott, Nucl. Phys. B 18B ( Proc. Suppl.) (1990) 29.

[14] A. Connes Noncommutative Geometry [Academic Press, 1994].

[15] J. Varilly and J.M. Gracia-Bondia, J. Geom. Phys. 12 (1993) 223.

[16] P. di Vecchia, K. Fabricius, G.C. Rossi and G. Veneziano, Nucl. Phys. B192 (1981) 392; M. Luscher Comm. Math. Phys. 85 (1982) 39.

[17] M. Göckeler, A.S. Kronfeld, M.L. Laursen, G. Schierholz and U.-J. Wiese, Phys. Lett. B233 (1989) 192.

[18] M. Teper, Nucl. Phys. B (Proc. Sup.) 20 (1991) 159. 
[19] R. Jackiw, Rev. Mod. Phys. 52 (1980) 661; M. Bos and V.P. Nair, Int. J. Mod. Phys. A5 (1990) 959; R. Dijkgraaf and E. Witten, Comm. Math. Phys. 129 (1990) 393.

[20] A.V. Phillips and D.A. Stone Nucl. Phys. B (Proc. Supp.) 20 (1991), 28.

[21] D. Eliezer and G. Semenoff, in Quantum Topology, L.H. Kauffman, R.A. Baadhio eds. [World Scientific, 1993] p. 139.

[22] S.G. Rajeev, Phys. Rev. D 42 (1990) 2779.

[23] G. Bimonte, E. Ercolessi, G. Landi, F. Lizzi, G. Sparano and P. Teotonio-Sobrinho preprints hep-th/9507147 and hep-th/9507148, to appear in J. Geom. Phys..

[24] P.S. Aleksandrov, Combinatorial Topology, Vol. 1-3 [Greylock, 1960]; R.P. Stanley, Combinatorics, Vol. 1 [Woodsworth and Brooks, Advanced Books and Software, 1986]; P.J. Hilton and S. Wylie, Homology Theory, [Cambridge University Press, 1966].

[25] L. Bombelli, J. Lee, D. Meyer and R. Sorkin, Phys. Rev. Lett. 59 (1987) 521.

[26] P.J. Hilton and S. Wylie, in [24]; W.S. Massey, Homology and Cohomology Theory (Marcel Dekker, New York, 1978).

[27] J. Madore, An Introduction to Noncommutative Differential Geometry and its Physical Applications, LMS 206, 1995; A. Dimakis, F. Müller-Hoissen, J. Phys. A 26 (1994) 3159; J. Math. Phys. 35 (1994) 6703; A. Dimakis, F. Müller-Hoissen and T. Striker, J. Phys. A 26 (1993) 1927; quant-ph/9509014; A. Sitarz, hepth/9603121.

[28] A.P. Balachandran, G. Bimonte, G. Marmo and A. Simoni, Nucl. Phys. B446 (1995) 299.

[29] A. Dimakis, F. Müller-Hoissen and T. Striker, in [27].

[30] H.B. Lawson and M.-L. Michelsohn, Spin Geometry [Princeton University Press, 1989].

[31] Cf. S. Coleman, $1 / N$ in Aspects of Symmetry, Selected Erice Lectures [Cambridge University Press, 1985].

[32] G. t'Hooft, Phys. Rep. 142 (1986) 257.

[33] A. Stern et al. , in preparation. 